Research Article

\title{
The Phenomenon of Bistable Phase Difference Intervals in the Times-Frequency Vibration Synchronization System Driven by Two Homodromy Exciters
}

\author{
Lingxuan Li (iD) and Xiaozhe Chen (D) \\ Department of Mechanical and Power Engineering, Northeastern University at Qinhuangdao, China, 066004 \\ Correspondence should be addressed to Xiaozhe Chen; xzchen1986@163.com
}

Received 12 November 2019; Accepted 22 January 2020; Published 31 March 2020

Guest Editor: Lazaros Moysis

Copyright (c) 2020 Lingxuan Li and Xiaozhe Chen. This is an open access article distributed under the Creative Commons Attribution License, which permits unrestricted use, distribution, and reproduction in any medium, provided the original work is properly cited.

\begin{abstract}
A vibration system with two homodromy exciters operated in different rotational speed is established to investigate whether the phenomenon of bistable phase difference intervals exists in the times-frequency vibration synchronization system. Some constructive conclusions are proposed. (1) By introducing an average angular velocity perturbation parameter $\varepsilon_{0}$ and two sets of phase difference perturbation parameters and $\varepsilon_{2}$, the frequency capture criterion and the necessary criteria for realizing the timesfrequency vibration synchronization are derived. The corresponding stability analysis is carried out. (2) By the theoretical analysis and experiments, it is verified that the times-frequency vibration synchronization system exists the phenomena of bistable phase difference interval. That is, the phase differences between the two homodromy exciters are stable around 180 degrees when they are located at a short distance; the antiphase synchronization phenomenon appears. On the contrary, they are stable around 0 degrees at the in-phase synchronization state. (3) Because of the two homodromy exciters operating in the different rotational speed, the vibration system obtains relatively complex compound motion trajectories; the corresponding application is investigated by adding a feeding material chamber. The times-frequency vibration synchronization system can be used to design the vibration mill for reducing its low-energy zone and developing chaotic mixing equipment for obtaining a better mixing effect.
\end{abstract}

\section{Introduction}

Synchronization can be explained as the adjustment of oscillating objects due to their weak interaction. As early as in the 17th century, because Huygens studied the coupling phenomenon of pendulum clocks, the phenomenon of inphase synchronization and antiphase synchronization was observed [1]. In the last century, Blekhman repeated this experiment, and the phenomenon was further observed and explained in theory. Later, Kozlov et al. found two van der Pol-Duffing oscillators with nonlinear coupling which can also realize the bistable phase synchronization [2]. The phenomena of bistable phase synchronization in nature have attracted more and more researchers to study deeply, such as active Nambu mechanics system [3], inhibitory coupled bursting neurons system [4], and coupled systems of piecewise constant oscillators [5].
Blekhman and Lurie have used the Poincare-Lyapunov small parameter method to study the vibration system composed of double eccentric rotors in 1953, pointing out that the system could realize self-synchronization and proving the system's motion stability $[6,7]$. Since then, the synchronization theory in the mechanical system has been studied. Recent studies have shown that the vibration system also has double phase synchronization phenomena, which mainly exist in four forms: (1) the bistable phase synchronization phenomenon is natural to appear in some vibration systems with double vibration mass or multimass $[8,9]$, including some rotor-pendulum systems $[10,11]$; (2) some vibration systems have the in-phase and antiphase synchronization phenomena when they operate in a subresonance or a superresonance state, respectively $[12,13] ;$ (3) the event of bistable phase difference intervals also exists in some near-resonance nonlinear vibration synchronous 
systems [8, 10]; (4) it is more popularly known in the vibration synchronization system with two homodromy exciters located at a single vibration mass body [14-16], including rotor-pendulum systems [10] and two eccentric rotors with a common rotational axis system $[17,18]$. The inphase synchronization phenomenon between the two homodromy exciters appears when they are located at a relatively far distance, i.e., the stable phase difference interval is $\left(-90^{\circ}, 90^{\circ}\right)$. On the contrary, the antiphase phenomenon appears and the steady interval is $\left(90^{\circ}, 270^{\circ}\right)$. In this condition, the system operates in the swing state, it has little engineering application in a general way. Therefore, the inphase synchronization phenomenon gains more traction compared with antiphase synchronization. Meanwhile, two or more exciters operating in the in-phase synchronization state can make the exciting force superimposition, which is possible to make the system gain a larger vibration amplitude with linear or near-elliptic motion trajectories.

In actuality, the structure of the system with two or more than two vibration masses is complex. Because of the limiting minimum natural frequency of the supporting springs, the rotational speed of the exciters is impossible to run at a relatively high speed, which makes the subresonance system not obtain a more significant exciting force even its structure is more complicated. Thus, the in-phase vibration synchronization system is usually designed as a single vibration mass with two homodromy exciters located at a far distance and operates in a superresonance state. But, the more realistic question is, just as mentioned above, obtaining a stable synchronization state for a system relies on the adjustment of oscillating objects due to their weak interaction. The longer distance between the two exciters means the more weak interaction; it is unfavorable for a mechanical system. At the same time, the impulse loads from mineral and some other external factors can easily disturb the weak interaction, which makes the system change violently to deviate from the normal in-phase synchronization state. Thus, for obtaining a stable synchronization state, it is necessary to design the vibration system with a relatively shorter distance between the two exciters for achieving a robustness state. Now that the vibration system powered by the same frequency supply cannot obtain the desired motion trajectories quickly, what can we do to realize the engineering requirements and protect the system from this kind of vulnerability? Maybe a times-frequency synchronization system is the right choice. Some vibration machines rely on gear unit or other speed change mechanism unit to achieve the synchronization state, such as double-frequency vibration compacting machine, vibrating feeder, multiaxis inertia table concentrator, and screening machine [19, 20]. The forced synchronization by rigid coupling causes even more serious problems especially in cracks of the mechanical structure, makes the system more complex, and decreases system reliability and stability. Therefore, the timesfrequency vibration synchronization theory may take on even more importance.

By the symmetric layout of four exciters in a vibration system of plane motion, the phenomenon of tripling frequency vibration synchronization is observed and investigated by Inoue and Araki in the 1970s [21]. It is a pity that the study did not continue to carry out related research and experiments on this basis. Subsequently, in the 1980s, Wen had pointed out that some nonlinear systems could achieve the times-frequency synchronization state. The system designed by Wen has two vibration masses; each one has two degrees of freedom, supported by piecewise nonlinear springs [19]. On the one hand, this system has a relatively complex mechanical structure; on the other hand, it rests in the conceptual phases of product design and the reliability cannot be proved by experiments.

The difficulty in the design of the times-frequency vibration synchronization system is just as the one pointed out by Wen; it is more challenging to implement the high-order harmonics and the subharmonic times-frequency capture than the fundamental frequency capture because of the smaller frequency capture interval [19]. Recently, a timesfrequency vibration system has been investigated by Jia, who gets a stable state by way of controlled synchronization [22]. If we can control the system based on the optimal structure parameters of the mechanical model, it will raise the efficiency, enhance the stability and the robustness, and decrease the energy loss by forcing control of the system. Thus, it is necessary and urgent to investigate the times-frequency vibration system to develop the theory of times-frequency synchronization and give out the critical structural parameters.

In our previous research, a vibration system with two exciters rotating in opposite directions is investigated. The condition of times-frequency vibration synchronization and its stability criterion are obtained, which is also proved by experimental studies. It shows that the system only exhibits in-phase vibration synchronization phenomenon [23]. In this study, we mainly investigate the vibration system with two homodromy exciters operating at different rotational speeds and find out whether the system exhibits bistable phase difference intervals and gives out the critical structural parameters and corresponding criteria. Furthermore, the engineering application of times-frequency vibration synchronization is also explored by adding a feeding material chamber to observe the motion trajectories of particle materials.

\section{Dynamics Equation and Steady-State Response of the System}

2.1. Dynamics Equation of the System. The typical model of the vibration synchronization system exciting by two homodromy exciters is shown in Figure 1, where oxy is the fixed coordinate system, $o^{\prime} x^{\prime} y^{\prime}$ is the moving coordinate system, $m$ is the mass of the rigid frame, $o$ is the mass center of the whole vibration system, $k_{x}, k_{y}$ and $c_{x}, c_{y}$ are the stiffness and damping of the system in $x, y$ direction, respectively. $m_{i}$ and $r_{i}$ are the mass and eccentric radius of the eccentric block of the exciter $i(i=1,2)$, respectively. $\dot{\varphi}_{i}$ and $\varphi_{i}$ are the instantaneous angular velocity and the angle of rotation relative to the starting point of the exciter $i(i=1,2)$, respectively. $l_{i}$ and $\beta_{i}$ are the distance from the axis of the exciter $i(i=1,2)$ to the mass center $o$ of the system and the 


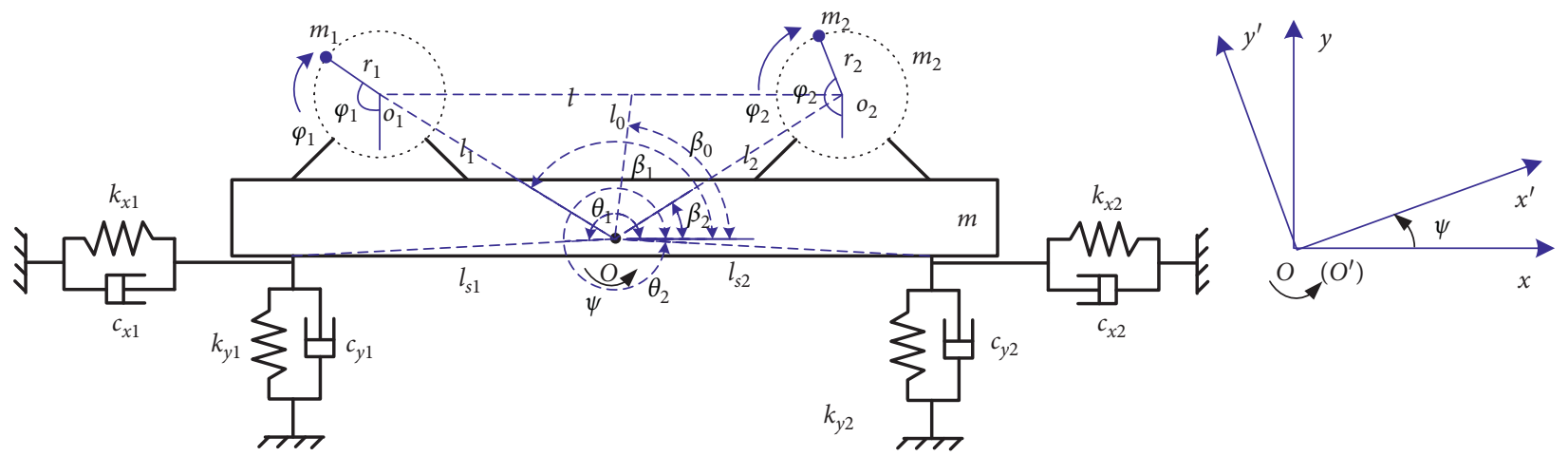

FIGURE 1: Dynamic model of the vibration system driven by two homodromy exciters.

angle between its connection and the horizontal direction, respectively. $l_{s i}$ and $\theta_{i}(i=1,2)$ are the distance from the connecting point between the spring $i(i=1,2)$ and frame to point $o$ and the angle between its connection and the horizontal direction. $l_{0}$ is the distance from the composite mass center of the two exciters to the mass center of the system, $\beta_{0}$ is the angle between $l_{0}$ and the horizontal direction, and $l$ is the distance from the axis of exciter 1 to the axis of exciter 2. By the way, all the nomenclature of the physical parameters and the symbols of intermediate parameters are normalized and listed at the end of the article and Table 1 , respectively.

Considering that the angular displacement $\psi$ of the system is too small comparing with $\beta_{i}$ and $\theta_{i}(i=1,2)$, $o$ can be left out $o$ when calculating the kinetic energy of the system and the potential of the springs. Based on Figure 1, the
TABLE 1: Symbols of intermediate parameters.

\begin{tabular}{lc}
\hline Items & Types \\
\hline$r, r_{m}, r_{l}, l_{s}, l_{e}, \beta, \alpha, \alpha_{0}, \tilde{\alpha}, \eta, \omega_{m}, \omega_{m 0}, \delta, \Delta, \gamma, p, W$ & $\begin{array}{c}\text { Single } \\
\text { value }\end{array}$ \\
\hline$\mu_{i 1}, \mu_{i 2}, \gamma_{i 1}, \gamma_{i 2}, \tau_{n i}(i=x, y, \psi)$ & $\begin{array}{c}\text { Single } \\
\text { value }\end{array}$ \\
\hline$\chi_{i j,}^{\prime}, \chi_{i j}\left(i=1,2_{\text {and }} j=1,2\right)$ & $\begin{array}{c}\text { Single } \\
\text { value }\end{array}$ \\
\hline$h_{i}, u_{i}, \rho_{i}, D_{i}, L_{R i}, W_{s i 0}, W_{c i 0}, W_{s i s}, W_{s i c}, W_{c i c}, W_{c i s}(i=1,2)$ & $\begin{array}{c}\text { Single } \\
\text { value }\end{array}$ \\
\hline $\mathbf{E}_{i}, \mathbf{C}_{i}(i=0,1)$ & Matrix \\
\hline $\mathbf{P , Q}, \mathbf{G}, \mathbf{A} \mathbf{U} \mathbf{I}$ & Matrix \\
\hline
\end{tabular}

equation of kinetic energy, potential energy, and energy dissipate function of the system is given as follows:

$$
\begin{aligned}
T= & \frac{1}{2} m_{0}\left[\left(\dot{x}+l_{0} \dot{\psi} \sin \beta_{0}\right)^{2}+\left(\dot{y}-l_{0} \dot{\psi} \cos \beta_{0}\right)^{2}\right]+\frac{1}{2} J_{p} \dot{\psi}^{2}+\frac{1}{2} \sum_{i=1}^{2} J_{i} \dot{\varphi}_{i}^{2} \\
& +\frac{1}{2} \sum_{i=1}^{2} m_{i}\left\{\left[\dot{x}-l_{i} \dot{\psi} \sin \beta_{i}-r_{i} \dot{\varphi}_{i} \sin \varphi_{i}\right]^{2}+\left[\dot{y}-l_{i} \dot{\psi} \cos \beta_{i}+r_{i} \dot{\varphi}_{i} \cos \varphi_{i}\right]^{2}\right\}, \\
V= & \frac{1}{2} \sum_{i=1}^{2}\left[k_{x i}\left(x-\rho_{1} \psi \sin \theta_{i}\right)^{2}+k_{y i}\left(-y-\rho_{i} \psi \cos \theta_{i}\right)^{2}\right], \\
D_{0}= & \frac{1}{2} \sum_{i=1}^{2}\left[c_{x i}\left(\dot{x}-\rho_{i} \dot{\psi} \sin \theta_{i}\right)^{2}+c_{y i}\left(-\dot{y}-\rho_{i} \dot{\psi} \cos \theta_{i}\right)^{2}\right]+\frac{1}{2} c_{1} \dot{\varphi}_{1}^{2}+\frac{1}{2} c_{2} \dot{\varphi}_{2}^{2},
\end{aligned}
$$

where $J_{p}$ is the moment of inertia of the vibration system to its mass center $o, J_{i}$ is the moment of inertia of the eccentric mass of the exciter $i(i=1,2)$ to its mass center, and $c_{i}$ is the damping coefficient of the axes of the exciter $i(i=1,2)$.

Taking $q=\left[x, y, \psi, \varphi_{1}, \varphi_{2}\right]^{T}$ as the generalized coordinate, substitute the kinetic energy $T$, potential energy $V$, and energy dissipate function $D_{0}$ of the system into the Lagrange equation and consider the following relations:

$$
\begin{aligned}
& m_{0} l_{0} \sin \left(\beta_{0}+\pi\right)+\sum_{i=1}^{2} m_{i} l_{i} \sin \beta_{i}=0 \\
& m_{0} l_{0} \cos \left(\beta_{0}+\pi\right)+\sum_{i=1}^{2} m_{i} l_{i} \cos \beta_{i}=0
\end{aligned}
$$

Then, the differential equation of motion of the system is obtained: 


$$
\begin{gathered}
\ddot{x} \sum_{i=0}^{2} m_{i}+\dot{x} \sum_{i=1}^{2} c_{x i}+x \sum_{i=1}^{2} k_{x i}-\dot{\psi} \sum_{i=1}^{2} l_{s i} c_{x i} \sin \theta_{i}-\psi \sum_{i=1}^{2} l_{s i} k_{x i} \sin \theta_{i}=\sum_{i=1}^{2} m_{i} r_{i}\left(\dot{\varphi}_{i}^{2} \cos \varphi_{i}+\ddot{\varphi}_{i} \sin \varphi_{i}\right), \\
\ddot{y} \sum_{i=0}^{2} m_{i}+\dot{y} \sum_{i=1}^{2} c_{y i}+y \sum_{i=1}^{2} k_{y i}+\dot{\psi} \sum_{i=1}^{2} l_{s i} c_{y i} \cos \theta_{i}+\psi \sum_{i=1}^{2} l_{s i} k_{y i} \cos \theta_{i}=\sum_{i=1}^{2} m_{i} r_{i}\left(\dot{\varphi}_{i}^{2} \sin \varphi_{i}+\ddot{\varphi}_{i} \cos \varphi_{i}\right), \\
\ddot{\psi}\left(J_{p}+\sum_{i=0}^{2} m_{i} l_{i}^{2}\right)+\dot{\psi} \sum_{i=1}^{2} l_{s i}^{2}\left(c_{x i} \sin ^{2} \theta_{i}+c_{y i} \cos ^{2} \theta_{i}\right)+\psi \sum_{i=1}^{2} l_{s i}^{2}\left(k_{x i} \sin ^{2} \theta_{i}+k_{y i} \cos ^{2} \theta_{i}\right)-\dot{x} \sum_{i=1}^{2} l_{s i} c_{x i} \sin \theta_{i} \\
+\dot{y} \sum_{i=1}^{2} l_{s i} c_{y i} \cos \theta_{i}-x \sum_{i=1}^{2} l_{s i} k_{x i} \sin \theta_{i}+y \sum_{i=1}^{2} l_{s i} k_{y i} \cos \theta_{i}=\sum_{i=1}^{2} m_{i} r_{i} l_{i}\left[\dot{\varphi}_{i}^{2} \sin \left(\varphi_{i}-\beta_{i}\right)-\ddot{\varphi}_{i} \cos \left(\varphi_{i}-\beta_{i}\right)\right], \\
J_{1} \ddot{\varphi}_{1}+c_{1} \dot{\varphi}_{1}-m_{1} r_{1}\left[\ddot{y} \cos \varphi_{1}-\ddot{x} \sin \varphi_{1}+l_{i} \ddot{\psi} \cos \left(\varphi_{1}-\beta_{1}\right)+l_{i} \psi^{2} \sin \left(\varphi_{1}-\beta_{1}\right)\right]=L_{e 1}, \\
J_{2} \ddot{\varphi}_{2}+c_{2} \dot{\varphi}_{2}-m_{2} r_{2}\left[\ddot{y} \cos \varphi_{2}-\ddot{x} \sin \varphi_{2}-l_{i} \ddot{\psi} \cos \left(\varphi_{2}-\beta_{2}\right)-l_{i} \psi^{2} \sin \left(\varphi_{2}-\beta_{2}\right)\right]=L_{e 2},
\end{gathered}
$$

where $J_{i}$ is the moment of inertia of the exciter $i(i=1,2)$ and $L_{e i}$ is the output torque of the motor which is driving the exciter $i(i=1,2)$.

2.2. Steady-State Response of the System. For a timesfrequency vibration system, the angular velocities of two exciters rotating in the same direction have definite multiple relations. Assume $n$ is the multiple relationships of rotational speed between the two exciters and $T_{0}$ is the single cycle of the high-speed exciter. Considering the multiple relationships in a common period $n T_{0}$, the angle of rotation should be $2 n \pi$ and $2 \pi$, respectively. Suppose the average phase of two exciters in steady-state is $\varphi$ and the average phase difference between the two exciters is $\alpha$. The phase relations in a common period between the two exciters can be represented by Figure 2 and the following equation:

$$
\begin{aligned}
& \varphi_{1}=n\left(\varphi+\alpha_{1}\right), \\
& \varphi_{2}=\varphi+\alpha_{2},
\end{aligned}
$$

where the steady-state phase difference of two exciters in its every single cycle is $\alpha_{1}$ and $\alpha_{2}$, respectively. From Figure 2 and equation (4), it can be concluded that the average steady-state phase difference of two exciters in a single common period is as follows:

$$
\alpha=n \alpha_{1}-\alpha_{2} .
$$

In this case, when the system is in a steady-state, the angular velocity $\dot{\varphi}_{i}$ of the eccentric block of exciter $i(i=1,2)$ should be constant; the relation between steady angular velocity and angular acceleration of the two exciters is as follows:

$$
\begin{aligned}
& \dot{\varphi}_{1}=n \dot{\varphi}_{2}=n \dot{\varphi} \approx n \omega_{m}, \\
& \ddot{\varphi}_{i} \approx 0, \quad(i=1,2) .
\end{aligned}
$$

Usually, in the same type of vibration system where the majority of models of the exciters are interchangeable, we only need to adjust the participating mass of the eccentric block to obtain the required exciting forces, that is, we can assume $r_{2}=r_{1}=r, m_{2}=\eta m_{r}$, and $m_{1}=m_{r}$. Considering the group arrangement of the same springs and the symmetrical arrangement of the exciters, we can get the following relations: $k_{x 1}=k_{x 2}=k_{x} / 2, k_{y 1}=k_{y 2}=k_{y} / 2, c_{x 1}=c_{x 2}=c_{x} / 2, c_{y 1}=$ $c_{y 2}=c_{y} / 2, l_{s 1}=l_{s 2}=l_{s}, \beta_{1}=\pi-\beta, \beta_{2}=\beta, \theta_{1} \approx \pi, \theta_{2} \approx 2 \pi$, and $l_{1}=l_{2}=l / 2 \cos \beta$. In this case, the first three equations in equation (3) can be simplified to

$$
\begin{aligned}
M \ddot{x}+f_{x} \dot{x}+k_{x} x & =m_{r} \dot{\varphi}^{2} r\left[\cos \left(n \varphi+n \alpha_{1}\right)+\eta \cos \left(\varphi+\alpha_{2}\right)\right], \\
M \ddot{y}+f_{y} \dot{y}+k_{y} y & =m_{r} \dot{\varphi}^{2} r\left[\sin \left(n \varphi+n \alpha_{1}\right)+\eta \sin \left(\varphi+\alpha_{2}\right)\right], \\
J \ddot{\psi}+\left(l_{s}^{2} c_{y}\right) \dot{\psi}+\left(l_{s}^{2} k_{y}\right) \psi & =m_{r} \dot{\varphi}^{2} r l\left[\sin \left(n \varphi+n \alpha_{1}-\beta\right)-\eta \sin \left(\varphi+\alpha_{2}+\beta\right)\right],
\end{aligned}
$$

where $M$ is the total mass of the vibration system, $M=m+\sum_{i=1}^{2} m_{i}$, and $J$ is the moment of inertia of the whole vibration system to the mass center of the system, $J=J_{p}+m l_{0}^{2}+\sum_{i=1}^{2} m_{i} l_{i}^{2}$. For a vibration system, the supporting positions of the spring can profoundly influence the dynamic characteristics of the whole system. As shown in equation (7), the location of the spring and the related parameters has a noticeable influence on the stiffness and damping of the system in the $\psi$ direction when it swings around the mass center of the body. Transforming equation (7) to the dimensionless form, we can conclude that

$$
\begin{aligned}
& \ddot{x}+2 \xi_{n x} \omega_{n x} \dot{x}+\omega_{n x}^{2} x=\dot{\varphi}^{2} r_{m} r\left[\cos \left(n \varphi+n \alpha_{1}\right)+\eta \cos \left(\varphi+\alpha_{2}\right)\right], \\
& \ddot{y}+2 \xi_{n y} \omega_{n y} \dot{y}+\omega_{n y}^{2} y=\dot{\varphi}^{2} r_{m} r\left[\sin \left(n \varphi+n \alpha_{1}\right)+\eta \sin \left(\varphi+\alpha_{2}\right)\right], \\
& \ddot{\psi}+2 \xi_{n \psi} \omega_{n \psi} \dot{\psi}+\omega_{n \psi}^{2} \psi=\dot{\varphi}^{2} r_{m} r \frac{r_{l}}{l_{e}}\left[\sin \left(n \varphi+n \alpha_{1}-\beta\right)-\eta \sin \left(\varphi+\alpha_{2}+\beta\right)\right],
\end{aligned}
$$




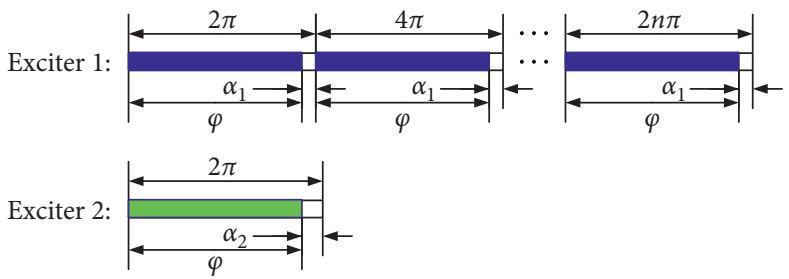

FIgURE 2: The phase relations in a common period between the two exciters.

where

$$
\begin{aligned}
\omega_{n x} & =\sqrt{\frac{k_{i}}{M}}, \\
\xi_{n x} & =\frac{c_{x}}{2 \sqrt{M k_{x}}}, \\
\omega_{n y} & =\sqrt{\frac{k_{y}}{M}}, \\
\xi_{n y} & =\frac{c_{y}}{2 \sqrt{M k_{y}}}, \\
\omega_{n \psi} & =\frac{l_{s}}{l_{e}} \omega_{n y}, \\
\xi_{n \psi} & =\frac{l_{s} c_{y}}{2 \sqrt{J k_{y}}}, \\
r_{l} & =\frac{l_{1}}{l_{e}}, \\
l_{e} & =\sqrt{\frac{J}{M}},
\end{aligned}
$$

The damping effect on the amplitude of the vibration system can be neglected because the damping ratio of the system is too small. Furthermore, the steady-state response of the system can be obtained by using the superposition principle as follows:

$$
\begin{aligned}
x= & -r_{m} r\left[\frac{1}{\mu_{x 1}} \cos \left(n \varphi+n \alpha_{1}+\gamma_{x 1}\right)+\frac{\eta}{\mu_{x 2}} \cos \left(\varphi+\alpha_{2}+\gamma_{x 2}\right)\right], \\
y= & -r_{m} r\left[\frac{1}{\mu_{y 1}} \sin \left(n \varphi+n \alpha_{1}+\gamma_{y 1}\right)+\frac{\eta}{\mu_{y 2}} \sin \left(\varphi+\alpha_{2}+\gamma_{y 2}\right)\right], \\
\psi= & -r_{m} r \frac{r_{l}}{l_{e}}\left[\frac{1}{\mu_{\psi 1}} \sin \left(n \varphi+n \alpha_{1}-\beta+\gamma_{\psi 1}\right)\right. \\
& \left.-\frac{\eta}{\mu_{\psi 1}} \sin \left(\varphi+\alpha_{2}+\beta+\gamma_{\psi 2}\right)\right]
\end{aligned}
$$

where

$$
\begin{aligned}
& \mu_{i 1}=1-\left(\frac{\tau_{n i}}{n}\right)^{2}, \\
& \mu_{i 2}=1-\tau_{n i}^{2}, \\
& \gamma_{i 1}=\arctan \frac{2 \xi_{n i} \tau_{n i}}{n \mu_{i 1}}, \\
& \gamma_{i 2}=\arctan \frac{2 \xi_{n i} \tau_{n i}}{\mu_{i 2}}, \\
& \tau_{n i}=\frac{\omega_{n i}}{\omega_{m}},
\end{aligned}
$$

$$
i=x, y, \psi \text {. }
$$

As mentioned in related references, it is noteworthy that the operating frequency of the superresonance vibration system is more than three times that of its natural frequency. For the multifrequency vibration machine, because of the limitation of the maximum speed of the exciting motor and the natural frequency of the supporting springs, the rotation speed of the low-speed exciter of the multifrequency vibration system can quickly fall near the natural frequency of the system. At this time, according to the phase-frequency characteristics of the vibration system, we can get that $\gamma_{i 2} \approx \pi / 2,(i=x, y, \psi)$. The trajectories of this kind of nearresonant machine are more complex and should be avoided as much as possible.

\section{Vibration Synchronization Conditions of the Times-Frequency Vibration System}

3.1. Frequency Capture Equation of the Times-Frequency Vibration System. The small perturbation parameter $\varepsilon_{0}(t)$ of average speed $\dot{\varphi}$ and the small phase perturbation parameters $\varepsilon_{1}(t)$ and $\varepsilon_{2}(t)$ of the two exciters are introduced when the system operates in a steady-state. In this case, the speed and acceleration of the two exciters in equation (6) can be expressed more accurately as follows:

$$
\begin{aligned}
& \dot{\varphi}_{1}=n\left(\dot{\varphi}+\dot{\alpha}_{1}\right)=n \omega_{m}\left[1+\varepsilon_{0}(t)+\varepsilon_{1}(t)\right], \\
& \dot{\varphi}_{2}=\dot{\varphi}+\dot{\alpha}_{2}=\omega_{m}\left[1+\varepsilon_{0}(t)+\varepsilon_{1}(t)\right], \\
& \ddot{\varphi}_{1}=n \omega_{m}\left[\dot{\varepsilon}_{0}(t)+\dot{\varepsilon}_{1}(t)\right], \\
& \ddot{\varphi}_{2}=\omega_{m}\left[\dot{\varepsilon}_{0}(t)+\dot{\varepsilon}_{2}(t)\right] .
\end{aligned}
$$


According to the requirements of the last two equations in equation (3), the required derivatives in $x, y$, and $\psi$ directions are easily worked out from the time-domain response of equation (10). Then, the relations in equation (12) should be considered. After substituting them into equation (10), the average values are obtained by integrating them in a common period. As known from equation (3), the average angular velocity should be constant when the system operates in a steady state, that is, when calculating the average values by integrals, there must be

$$
\begin{aligned}
& \dot{\bar{\varepsilon}}_{0}=\int_{t}^{t+n T_{0}} \dot{\varepsilon}_{0}(t) \mathrm{d} t=0, \\
& \bar{\varepsilon}_{0}=\int_{t}^{t+n T_{0}} \varepsilon_{0}(t) \mathrm{d} t=0 .
\end{aligned}
$$

Then, the following equations can be obtained:

$$
\begin{gathered}
J_{1} n \omega_{m} \dot{\bar{\varepsilon}}_{1}+n \omega_{m} c_{1}\left(1+\bar{\varepsilon}_{1}\right)=L_{e 1}-\frac{1}{2} m_{r} \omega_{m} r^{2} r_{m}\left\{\left(\chi_{11}^{\prime} \dot{\bar{\varepsilon}}_{1}+\chi_{12}^{\prime} \dot{\bar{\varepsilon}}_{2}\right)+2 \omega_{m}\left(\chi_{11} \bar{\varepsilon}_{1}+\chi_{12} \bar{\varepsilon}_{2}\right)+h_{1}\right\}, \\
J_{2} \omega_{m} \dot{\bar{\varepsilon}}_{2}+\omega_{m} c_{2}\left(1+\bar{\varepsilon}_{2}\right)=L_{e 2}-\frac{1}{2} m_{r} \omega_{m} r^{2} r_{m}\left[\left(\chi_{21}^{\prime} \dot{\bar{\varepsilon}}_{1}+\chi_{22}^{\prime} \dot{\bar{\varepsilon}}_{2}\right)+2 \omega_{m}\left(\chi_{21} \bar{\varepsilon}_{1}+\chi_{22} \bar{\varepsilon}_{2}\right)+h_{2}\right],
\end{gathered}
$$

where

$$
\begin{aligned}
& \alpha=n \alpha_{1}-\alpha_{2}, \\
& \chi_{11}^{\prime}=-n W_{c 10}, \\
& \chi_{12}^{\prime}=-\eta\left[\left(W_{s 2 s}-W_{s 2 c}\right) \sin \alpha+\left(W_{c 2 s}+W_{c 2 c}\right) \cos \alpha\right], \\
& \chi_{21}^{\prime}=n \eta\left[\left(W_{s 1 s}+W_{s 1 c}\right) \sin \alpha+\left(W_{c 1 s}-W_{c 1 c}\right) \cos \alpha\right], \\
& \chi_{22}^{\prime}=-\eta^{2} W_{c 20}, \\
& \chi_{11}=n^{2} W_{s 10}, \\
& \chi_{12}=\eta\left[-\left(W_{c 2 s}+W_{c 2 c}\right) \sin \alpha+\left(W_{s 2 s}-W_{s 2 c}\right) \cos \alpha\right], \\
& \chi_{21}=n^{2} \eta\left[\left(W_{c 1 c}-W_{c 1 s}\right) \sin \alpha+\left(W_{s 1 s}+W_{s 1 c}\right) \cos \alpha\right], \\
& \chi_{22}=\eta^{2} W_{s 20}, \\
& h_{1}=\omega_{m}\left[-\eta\left(W_{c 2 c}+W_{c 2 s}\right) \sin \alpha+\eta\left(W_{s 2 c}-W_{s 2 s}\right) \cos \alpha+n^{2} W_{s 10}\right], \\
& h_{2}=\omega_{m} \eta\left[n^{2}\left(W_{c 1 c}-W_{c 1 s}\right) \sin \alpha+n^{2}\left(W_{s 1 s}+W_{s 1 c}\right) \cos \alpha+\eta W_{s 20}\right], \\
& W_{s i 0}=\frac{\sin \gamma_{x i}}{\mu_{x i}}+\frac{\sin \gamma_{y i}}{\mu_{y i}}+r_{l}^{2} \frac{\sin \gamma_{\psi i}}{\mu_{\psi i}}, \\
& W_{c i 0}=\frac{\cos \gamma_{x i}}{\mu_{x i}}+\frac{\cos \gamma_{y i}}{\mu_{y i}}+r_{l}^{2} \frac{\cos \gamma_{\psi i}}{\mu_{\psi i}}, \quad i=1,2, \\
& W_{s i s}=\frac{\sin \gamma_{x i}}{\mu_{x i}}+\frac{\sin \gamma_{y i}}{\mu_{y i}}-r_{l}^{2} \frac{\sin \gamma_{\psi i}}{\mu_{\psi i}} \cos 2 \beta, \\
& W_{s i c}=r_{l}^{2} \frac{\cos \gamma_{\psi i}}{\mu_{\psi i}} \sin 2 \beta, \quad i=1,2, \\
& \mu_{x i}
\end{aligned}
$$


Because the rotational inertia of the axis of the exciter is much smaller than that of the eccentric mass on the axis, it can be neglected. Thus, the moment of inertia of the exciter is $J_{i} \approx m_{i} r_{i}^{2}(i=1,2)$. Considering the relation between the motor output torque $L_{e 0 i}$ of the exciter $i(i=1,2)$ and its stiffness coefficients $k_{e 0 i}$ of the angular velocity at a certain speed $\omega_{m}$,

$$
L_{e i}=L_{e 0 i}-k_{e 0 i} \bar{\varepsilon}_{i}, \quad i=1,2 .
$$

The frequency capture equation of the times-frequency vibration synchronization system can be obtained by substituting them into equation (14) and sorting it out as a matrix form. We have

$$
\mathbf{E}_{1} \cdot\left|\begin{array}{l}
\dot{\bar{\varepsilon}}_{1} \\
\dot{\bar{\varepsilon}}_{2}
\end{array}\right|=\mathbf{C}_{1} \cdot\left|\begin{array}{l}
\bar{\varepsilon}_{1} \\
\bar{\varepsilon}_{2}
\end{array}\right|+\left|\begin{array}{l}
u_{1} \\
u_{2}
\end{array}\right|
$$

where

$$
\begin{aligned}
& \mathbf{E}_{1}=\frac{1}{2} m_{r} \omega_{m} r^{2} r_{m}\left|\begin{array}{cc}
n\left(\frac{2}{r_{m}}-W_{c 10}\right) & -\eta\left[\left(W_{s 2 s}-W_{s 2 c}\right) \sin \alpha+\left(W_{c 2 s}+W_{c 2 c}\right) \cos \alpha\right] \\
n \eta\left[\left(W_{s 1 s}+W_{s 1 c}\right) \sin \alpha+\left(W_{c 1 s}-W_{c 1 c}\right) \cos \alpha\right] & \eta\left(\frac{2}{r_{m}}-\eta W_{c 20}\right)
\end{array}\right|, \\
& \mathbf{C}_{1}=m_{r} \omega_{m}^{2} r^{2} r_{m}\left|\begin{array}{cc}
-n\left(n W_{s 10}+\tau_{1}\right) & \eta\left[\left(W_{c 2 s}+W_{c 2 c}\right) \sin \alpha+\left(W_{s 2 c}-W_{s 2 s}\right) \cos \alpha\right] \\
-n^{2} \eta\left[\left(W_{c 1 c}-W_{c 1 s}\right) \sin \alpha+\left(W_{s 1 s}+W_{s 1 c}\right) \cos \alpha\right] & -\left(\eta^{2} W_{s 20}+\tau_{2}\right)
\end{array}\right|, \\
& u_{1}=L_{e 01}-n f_{1} \omega_{m}-\frac{1}{2} n^{2} m_{r} \omega_{m}^{2} r^{2} r_{m} W_{s 10}+\frac{1}{2} \eta m_{r} \omega_{m}^{2} r^{2} r_{m}\left[\left(W_{c 2 c}+W_{c 2 s}\right) \sin \alpha+\left(W_{s 2 c}-W_{s 2 s}\right) \cos \alpha\right] \text {, } \\
& u_{2}=L_{e 02}-f_{2} \omega_{m}-\frac{1}{2} \eta^{2} m_{r} \omega_{m}^{2} r^{2} r_{m} W_{s 20}-\frac{1}{2} n^{2} \eta m_{r} \omega_{m}^{2} r^{2} r_{m}\left[\left(W_{c 1 c}-W_{c 1 s}\right) \sin \alpha+\left(W_{s 1 s}+W_{s 1 c}\right) \cos \alpha\right] \text {, } \\
& \tau_{1}=\frac{k_{e 01}+c_{1} \omega_{m}}{m_{r} \omega_{m}^{2} r^{2}} \\
& \tau_{2}=\frac{k_{e 02}+c_{2} \omega_{m}}{m_{r} \omega_{m}^{2} r^{2}}
\end{aligned}
$$

3.2. Necessary Conditions for Realizing Times-Frequency Vibration Synchronization. For the dynamic system shown in equation (3), to make the system running stability, as mentioned before, the first three equations of equation (3) must have a steady solution. Furthermore, the last two equations of equation (3) also must be stable under the perturbations, that is, for the frequency capture equation of the times-frequency vibration synchronization system shown in equation (17), there must be $\dot{\bar{\varepsilon}}_{i}=0$ and $\bar{\varepsilon}_{i}=0(i=1,2)$. It means $u_{i}=0(i=1,2)$. Then, subtracting the two equations, the moment balance equations for the frequency capture of two exciters can be obtained:

$$
-\left[n^{2}\left(W_{c 1 c}-W_{c 1 s}\right)+W_{c 2 c}+W_{c 2 s}\right] \sin \alpha-\left[n^{2}\left(W_{s 1 c}+W_{s 1 s}\right)+W_{s 2 c}-W_{s 2 s}\right] \cos \alpha=\frac{L_{R 1}-L_{R 2}}{2 \eta m_{r} \omega_{m}^{2} r^{2} r_{m}}
$$

where

$$
\begin{aligned}
& L_{R 1}=L_{e 01}-n c_{1} \omega_{m}-\frac{1}{2} n^{2} m_{r} \omega_{m}^{2} r^{2} r_{m} W_{s 10}, \\
& L_{R 2}=L_{e 02}-c_{2} \omega_{m}-\frac{1}{2} \eta^{2} m_{r} \omega_{m}^{2} r^{2} r_{m} W_{s 20} .
\end{aligned}
$$

It is noteworthy that the system damping of the vibration synchronization equipment is small. For the vibration system which does not work in the subresonance or superresonance state, the value of phase difference angle of the time-domain displacement responses lagging the exciting loads approximates to zero in the low-frequency working condition, which is approximate to $\pi$ in the high-frequency working condition. Therefore, it means that $W_{s i s}, W_{s i 0}$, and $W_{c i s}$ are much less than $W_{s i c}, W_{c i 0}$, and $W_{c i c}(i=1,2)$ in the frequency capture equation. Then, equation (19) can be simplified to

$$
-\left(n^{2} W_{c 1 c}+W_{c 2 c}\right) \sin \alpha-\left(n^{2} W_{s 1 c}+W_{s 2 c}\right) \cos \alpha=\frac{2\left(L_{R 1}-L_{R 2}\right)}{\eta m_{r} \omega_{m}^{2} r^{2} r_{m}} .
$$


Furthermore, we have

$$
\begin{aligned}
\sin (\alpha+\delta) & =\frac{1}{D}, \\
\alpha & =\arcsin \left(\frac{1}{D}\right)-\delta,
\end{aligned}
$$

where

$$
\delta= \begin{cases}\arctan \left(\frac{n^{2} W_{s 1 c}+W_{s 2 c}}{n^{2} W_{c 1 c}+W_{c 2 c}}\right), & n^{2} W_{c 1 c}+W_{c 2 c}<0, \\ -\pi+\arctan \left(\frac{n^{2} W_{s 1 c}+W_{s 2 c}}{n^{2} W_{c 1 c}+W_{c 2 c}}\right), & n^{2} W_{c 1 c}+W_{c 2 c} \geq 0,\end{cases}
$$

and $D$ is a synchronization performance index with a value of

$$
D=\frac{1 / 2 m_{r} \omega_{m}^{2} r^{2} r_{m} \eta \sqrt{\left(n^{2} W_{s 1 c}+W_{s 2 c}\right)^{2}+\left(n^{2} W_{c 1 c}+W_{c 2 c}\right)^{2}}}{T_{r 1}-T_{r 2}} .
$$

If $|D|<1$, there are no solutions in equation (22), the stationary equation cannot be satisfied, the eccentric block of the two exciters does not have a stable phase difference, and the system cannot achieve a times-frequency synchronization vibration state. Therefore, the necessary condition for realizing times-frequency vibration synchronization of the vibration system is the synchronization performance index:

$$
|D| \geq 1 \text {. }
$$

\subsection{Stability Criterion of the Vibration Synchronization State. To analyze whether the system can run stably near the equilibrium point, the critical point is to check the global stability of the system at the equilibrium point. Moreover, we need to discuss whether the system is uniformly asymp- totically stable and exponentially stable at the equilibrium point.}

3.3.1. Equivalent Perturbed System. The Taylor expansion is used to linearize equation (17) at $\alpha=\alpha_{0}$ and $\omega_{m}=\omega_{m 0}$. Then, the equivalent linearized formula is obtained by taking into account that $W_{s i s}, W_{s i 0}$, are $W_{c i s}$ are far less than $W_{s i c}, W_{c i 0}$, and $W_{c i c}(i=1,2)$ :

$$
\mathbf{E}_{0} \cdot\left|\begin{array}{c}
\dot{\bar{\varepsilon}}_{1} \\
\dot{\bar{\varepsilon}}_{2}
\end{array}\right|=\mathbf{C}_{0} \cdot\left|\begin{array}{c}
\bar{\varepsilon}_{1} \\
\bar{\varepsilon}_{2}
\end{array}\right|+\mathbf{U}_{0}
$$

where

$$
\begin{aligned}
& \mathbf{E}_{0}=\left|\begin{array}{cc}
n \rho_{1} & \frac{1}{2} \eta\left(W_{s 2 c} \sin \alpha_{0}-W_{c 2 c} \cos \alpha_{0}\right) \\
\frac{1}{2} n \eta\left(W_{s 1 c} \sin \alpha_{0}-W_{c 1 c} \cos \alpha_{0}\right) & \rho_{2}
\end{array}\right|, \\
& \mathbf{C}_{0}=\omega_{m}\left|\begin{array}{cc}
-n \tau_{1} & \eta\left(W_{c 2 c} \sin \alpha_{0}+W_{s 2 c} \cos \alpha_{0}\right) \\
-n^{2} \eta\left(W_{c 1 c} \sin \alpha_{0}+W_{s 1 c} \cos \alpha_{0}\right) & -\tau_{2}
\end{array}\right|, \\
& \mathbf{U}_{0}=\frac{1}{2} \eta \omega_{m}\left(\alpha-\alpha_{0}\right)\left|\begin{array}{c}
-W_{s 2 c} \sin \alpha_{0}+W_{c 2 c} \cos \alpha_{0} \\
n^{2}\left(W_{s 1 c} \sin \alpha_{0}-W_{c 1 c} \cos \alpha_{0}\right)
\end{array}\right|, \\
& \rho_{1}=\frac{1}{r_{m}}-\frac{1}{2} W_{c 10}, \\
& \rho_{2}=\frac{\eta}{r_{m}}-\frac{\eta^{2}}{2} W_{c 20} .
\end{aligned}
$$

When $\mathbf{E}_{0}$ is invertible, the following equation should be satisfied:

$$
\begin{aligned}
\Delta= & n\left[\rho_{1} \rho_{2}-\frac{1}{4} \eta^{2}\left(W_{s 1 c} \sin \alpha_{0}-W_{c 1 c} \cos \alpha_{0}\right)\right. \\
& \left.\cdot\left(W_{s 2 c} \sin \alpha_{0}-W_{c 2 c} \cos \alpha_{0}\right)\right] \neq 0 .
\end{aligned}
$$

For the vibration system, the mass $m$ of the eccentric block of the exciter is far less than the mass $M$ of the whole vibration system, so $r_{m} \ll 1$. Besides, the vibration synchronization system working in the superresonance state is a typical low damped vibration system and the phase of the time-domain response solution is almost antiphase, different from that of the exciting load. Considering $r_{l}<7$ as discussed in $[24,25]$, we have $\rho_{1} \ll 1$ and $\rho_{2} \ll 1$ in equation (26). Furthermore, it means that $\Delta$ in equation (28) must be greater than 0 .

Then, a uniform model of the perturbed system of equation (26) can be given as

$$
\dot{x}=f(t, x)+g(t, x) \text {, }
$$

where 


$$
\begin{aligned}
f(t, x) & =\mathbf{A} x, \\
g(t, x) & =\mathbf{E}_{0}^{-1} \mathbf{U}_{0}, \\
\mathbf{A} & =\mathbf{E}_{0}^{-1} \mathbf{C}_{0}, \\
x & =\left[\begin{array}{ll}
\bar{\varepsilon}_{1} & \bar{\varepsilon}_{2}
\end{array}\right]^{T} .
\end{aligned}
$$

It is noteworthy that the remainder term $g(t, x)$ contains $\alpha$, which is dependent on the time-domain variable $t$, usually named as a perturbation factor of the phase difference. Although the changes of small parameters $\bar{\varepsilon}_{1}$ and $\bar{\varepsilon}_{2}$ will cause the change of $\alpha$, the equation of the remainder term has a definite upper limiting value and lower limiting value, that is, for all $t \geq 0$, there is a positive value $\gamma$ can make $\|g(t, x)\|_{2}<\gamma\|x\|_{2}$. In this case, according to [26], the term $g(t, x)$ can be treated as an additive term of the system $\dot{x}=f(t, x)$, which does not change the order and the uncertainty of the system.

3.3.2. Stability of the Uniform Perturbed System under Angular Velocity Perturbation. According to equation (29), when $x=0, g(t, 0)=0$, which means that the origin point of the perturbed system is an equilibrium point. In this case, the stability of the origin point can be analyzed as the stability of the perturbed system. That is to say, the problem of whether the times-frequency vibration system can achieve the vibration synchronization state can be directly treated as solving the stability of the system at the origin point under the condition of the angular velocity perturbation and the phase difference perturbation.

Firstly, considering the stability of the system under angular velocity perturbation parameters, i.e., let phase difference perturbation term $g(t, x)=0$, the system in equation (29) becomes a two-dimensional ordinary differential system. When the matrix A satisfies the criteria required by the Hurwitz matrix, i.e., all the eigenvalues of $\mathbf{A}$ having $\operatorname{Re} \lambda(\mathbf{A})<0$, the system will be asymptotically stable at the origin point and the zero solutions are asymptotically stable. In this case, the first-order subdeterminants matrix of the coefficient matrix $\mathbf{A}$ is less than 0 and its second-order subdeterminants matrix must be greater than 0 , that is,

$$
\begin{gathered}
\frac{n \omega_{m}}{\Delta}\left[\tau_{1} \rho_{2}+\frac{1}{2} n \eta^{2}\left(W_{c 1 c} \sin \alpha_{0}+W_{s 1 c} \cos \alpha_{0}\right)\right. \\
\left.\cdot\left(W_{s 2 c} \sin \alpha_{0}-W_{c 2 c} \cos \alpha_{0}\right)\right]>0, \\
\frac{n \omega_{m}^{2}}{\Delta}\left[\tau_{1} \tau_{2}+n \eta^{2}\left(W_{c 1 c} \sin \alpha_{0}+W_{s 1 c} \cos \alpha_{0}\right)\right. \\
\left.\cdot\left(W_{c 2 c} \sin \alpha_{0}+W_{s 2 c} \cos \alpha_{0}\right)\right]>0 .
\end{gathered}
$$

As shown in equation (17), the term $m \omega_{m}^{2} r^{2}$ in the denominator is the exciting force multiplied by the eccentric radius of the exciter. It is far less than the stiffness coefficients $k_{e 0 i}(i=1,2)$ of the angular velocity of the motor of the exciter [27]. At the same time, we note that $\rho_{2} \ll 1$. Therefore, the criteria in equation (31) can be satisfied. Furthermore, it means that the matrix $\mathbf{A}$ is a Hurwitz matrix and the system is asymptotically stable, the system is stable under angular velocity perturbation.

\subsubsection{Stability of the Uniform Perturbed System under Phase-} Difference Perturbation. As concluded in the previous section, the matrix $\mathbf{A}$ is a Hurwitz matrix, which is a prerequisite for the stability of the system under phase-difference perturbation. Since $\operatorname{Re} \lambda(\mathbf{A})<0$, we can assume $\mathbf{Q}=\mathbf{Q}^{T}>0$, which is satisfying the following Lyapunov equation:

$$
\mathbf{P A}+\mathbf{A}^{T} \mathbf{P}=-\mathbf{Q} .
$$

The Lyapunov theorem shows that the equation exhibits a unique solution, in which $\mathbf{P}$ is a positive Hermite matrix. In this case, the quadratic Lyapunov function $V(x)=x^{T} \mathbf{P} x$ must satisfy [26] the following condition:

$$
\frac{\partial V}{\partial x} \mathbf{A} x=-x^{T} \mathbf{Q} x \leq-\lambda_{\min }(\mathbf{Q})\|x\|_{2}^{2}
$$

Thus, the derivative of $V(x)$ along the trajectory of the perturbed system will satisfy

$$
\begin{aligned}
\dot{V}(t, x)= & \frac{\partial V}{\partial x} \mathbf{A} x+\frac{\partial V}{\partial x} g(t, x) \\
= & -x^{T} \mathbf{Q} x+2 x^{T} \mathbf{P} g(t, x) \leq-\lambda_{\min }(\mathbf{Q})\|x\|_{2}^{2} \\
& +2 x^{T} \mathbf{P} g(t, x),
\end{aligned}
$$

where $\lambda_{\text {min }}(\mathbf{Q})$ is the minimum eigenvalue of the matrix $\mathbf{Q}$. We should note that the first term of right-hand side in equation (34) is less than 0 because of $\mathbf{Q}=\mathbf{Q}^{T}>0$. Thus, $\dot{V}(t, x)$ returns a negative or positive value mainly depending on the positive or negative sign of the second term $2 x^{T} \mathbf{P} g(t, x)$.

Assume

$$
\mathbf{Q}=-p\left[\begin{array}{cc}
2 a_{21} & a_{11}+a_{22} \\
a_{11}+a_{22} & 2 a_{12}
\end{array}\right]-\left[\begin{array}{cc}
2 a_{11} & a_{12}+a_{21} \\
a_{12}+a_{21} & 2 a_{22}
\end{array}\right] \text {. }
$$

We can see that the second term $\mathbf{Q}$ in equation (35) is a symmetric matrix composed of $-\left(\mathbf{A}+\mathbf{A}^{T}\right)$. The first term is also a symmetric matrix obtained by the primary row transformation and then further combined similarly and then multiplied by $p$. Therefore, the necessary condition for $\mathbf{Q}=\mathbf{Q}^{T}>0$ is

$$
p>0 \text {. }
$$

By substituting $\mathbf{Q}$ into equation (32), we have

$$
\mathbf{P}=\left[\begin{array}{ll}
1 & p \\
p & 1
\end{array}\right]
$$

Then, we can conclude that the criterion of the symmetric matrix $\mathbf{P}$ and $\mathbf{Q}$ are positive definite matrixes is $0<p<1$. Furthermore, substituting the value of the matrix $\mathbf{P}$ for the last term of equation (34), we have 


$$
2 x^{T} \mathbf{P} g(t, x)=\eta \omega_{m 0}\left(\alpha-\alpha_{0}\right)\left[\begin{array}{ll}
x_{1} & x_{2}
\end{array}\right](\mathbf{G})_{2 \times 2}\left[-W_{s 2 c} \sin \alpha_{0}+W_{c 2 c} \cos \alpha_{0} n^{2}\left(W_{s 1 c} \sin \alpha_{0}-W_{c 1 c} \cos \alpha_{0}\right)\right]^{T},
$$

where

$$
\mathbf{G}=\left(\mathbf{P E}_{0}^{-1}\right)_{2 \times 2}=\frac{1}{\Delta}\left|\begin{array}{ll}
\rho_{2}-\frac{p}{2} n \eta\left(W_{s 1 c} \sin \alpha_{0}-W_{c 1 c} \cos \alpha_{0}\right) & p n \rho_{1}-\frac{1}{2} \eta\left(W_{s 2 c} \sin \alpha_{0}-W_{c 2 c} \cos \alpha_{0}\right) \\
p \rho_{2}-\frac{1}{2} n \eta\left(W_{s 1 c} \sin \alpha_{0}-W_{c 1 c} \cos \alpha_{0}\right) & n \rho_{1}-\frac{p}{2} \eta\left(W_{s 2 c} \sin \alpha_{0}-W_{c 2 c} \cos \alpha_{0}\right)
\end{array}\right|
$$
have

Calculating the determinant values of the matrix $\mathbf{G}$, we

$$
\begin{aligned}
& D_{1}=\frac{\rho_{2}-p / 2 n \eta\left(W_{s 1 c} \sin \alpha_{0}-W_{c 1 c} \cos \alpha_{0}\right)}{\Delta}>0, \\
& D_{2}=1-p^{2}>0 .
\end{aligned}
$$

As mentioned above, $\rho_{2} \gg 1$ and $1>p>0$; therefore, the matrix $\mathbf{G}$ is a positive definite matrix, its eigenvalues must be greater than 0 . According to the comparison relation of partial order in matrix theory [28],

$$
\lambda_{\min }(\mathbf{G}) \mathbf{I} \preceq \mathbf{G} \preceq \lambda_{\max }(\mathbf{G}) \mathbf{I} .
$$

Then, equation (38) can be further converted into the following:

$$
\begin{aligned}
2 x^{T} \mathbf{P} g(t, x) \leq & \lambda_{\min }(\mathbf{G})_{2 \times 2} \eta \omega_{m 0}\left(\alpha-\alpha_{0}\right) \\
& \cdot\left[\left(-W_{s 2 c} \sin \alpha_{0}+W_{c 2 c} \cos \alpha_{0}\right) x_{1}\right. \\
& \left.+n^{2}\left(W_{s 1 c} \sin \alpha_{0}-W_{c 1 c} \cos \alpha_{0}\right) x_{2}\right] .
\end{aligned}
$$

As the previous definition $x=\left[\begin{array}{ll}\bar{\varepsilon}_{1} & \bar{\varepsilon}_{2}\end{array}\right]^{T}, \widetilde{\alpha}=\alpha-\alpha_{0}$ has the same symbols as $n x_{1}-x_{2}$ because $\widetilde{\alpha}$ is an integral mean value point obtained by integrating the perturbation term $n \bar{\varepsilon}_{1}-\bar{\varepsilon}_{2}$ over time in a common period $n T_{0}$, that is,

$$
\widetilde{\alpha}\left(n x_{1}-x_{2}\right)>0 \text {. }
$$

$\widetilde{\alpha}, x_{1}$, and $x_{2}$ may vary continuously along with the time $t$; all possible contingencies need to be examined. The first condition, if $\tilde{\alpha}>0$, we have $x_{2}<n x_{1}$ according to equation (43). In this case, equation (42) can be written as

$$
\begin{aligned}
2 x^{T} \mathbf{P} g(t, x)< & \lambda_{\min }(\mathbf{G})_{2 \times 2} \eta \omega_{m 0} \widetilde{\alpha} x_{1} W \\
W= & \left(-W_{s 2 c} \sin \alpha_{0}+W_{c 2 c} \cos \alpha_{0}\right) \\
& +n^{3}\left(W_{s 1 c} \sin \alpha_{0}-W_{c 1 c} \cos \alpha_{0}\right) .
\end{aligned}
$$

For equation (44), $W \leq 0$ when $x_{1} \geq 0$; or $W \geq 0$ when $x_{1}<0$, the system can be stable.

The second condition is that when $\tilde{\alpha} \leq 0$, there is $x_{1} \leq x_{2} / n$. Correspondingly, equation (42) can be written as

$$
2 x^{T} \mathbf{P} g(t, x)<\lambda_{\min }(\mathbf{G})_{2 \times 2} \eta \omega_{m 0} \widetilde{\alpha} x_{2} n W .
$$

Obviously, for equation (45), $W \geq 0$ when $x_{2} \geq 0$; or $W \leq 0$ when $x_{2}<0$, the system is stable.

From the discussions in equations (44) and (45), it is known that if and only if $W$ is equal to 0 , the synchronization state of times-frequency vibration system can be made stable under phase difference perturbation. This is certainly true of that $W=0$ has solutions. Thus, the times-frequency system can operate in a steady state.

Actually, the boundaries with large margins are obtained by directly calculating $2 x^{T} \mathbf{P} g(t, x) \leq 0$ for such type of zero perturbation system. Even if the perturbation term $2 x^{T} \mathbf{P} g(t, x)$ of the perturbed system is greater than 0 , the derivative $V(t, x)$ along the trajectory of the perturbed system can also be calculated by using the general boundary [29]. That is,

$$
\begin{aligned}
\dot{V}(t, x)= & \frac{\partial V}{\partial x} \mathbf{A} x+\frac{\partial V}{\partial x} g(t, x)=-x^{T} \mathbf{Q} x+2 x^{T} \mathbf{P} g(t, x) \\
& \leq-\lambda_{\min }(\mathbf{Q})\|x\|_{2}^{2}+2 \lambda_{\max }(\mathbf{P}) \gamma\|x\|_{2}^{2} .
\end{aligned}
$$

The origin point can be globally exponentially stable when $\gamma<\lambda_{\min }(\mathbf{Q}) / 2 \lambda_{\max }(\mathbf{P})$. The maximum limit of $\gamma$ can be obtained by taking $\mathbf{Q}=\mathbf{I}$ in equation (46) [30].

To sum up, the stability of times-frequency vibration synchronization under angular velocity perturbation and phase difference perturbation are proved.

\section{Experimental Analysis and Theoretical Verification}

4.1. Analysis of the Empirical Phenomena. According to the mechanical model of the vibration system in Figure 1, an innovative platform is constructed as shown in Figure 3. It mainly includes a times-frequency vibration system, signal acquisition system, high-speed camera system, and some sensors. The structural parameters are uniform, and the relative property is quite strong; two same types of exciters are used, denoted by exciter 1 and exciter 2. Besides, another two exciters with equal mass distributions are also assembled on the frame of the system as balance weight during the test period. The main parameters of the exciters and the vibration system are listed in Tables 2 and 3, respectively. 


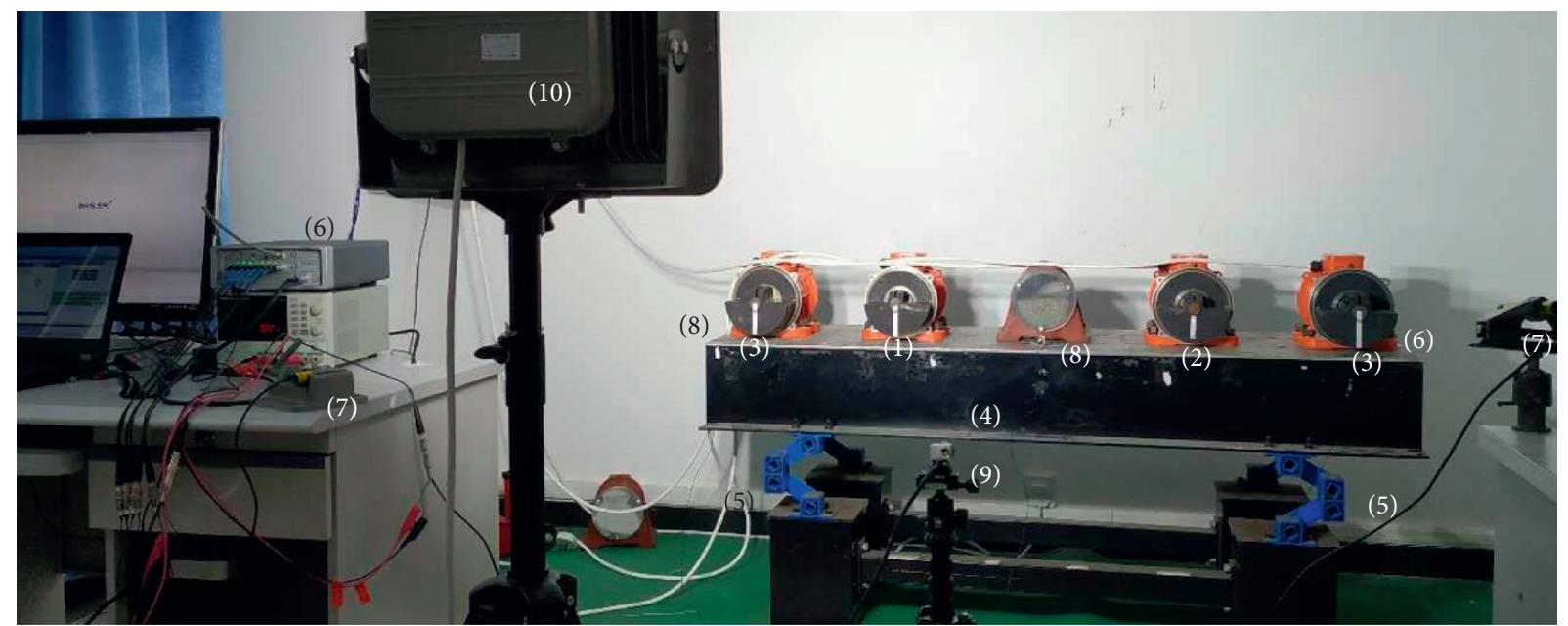

FIGURE 3: Times-frequency vibration synchronization experiment system. (1) Exciter 1, (2) exciter 2, (3) balance weight, (4) rigid frame of the system, (5) four springs with the type of ROSTA AB27, (6) signal acquisition system, (7) two ROLSs (remote optical laser sensors), (8) three triaxial accelerometers, (9) Basler acA1440-220uc camera, and (10) supplement light system for the camera.

TABLE 2: Main parameters of the exciters.

\begin{tabular}{lccc}
\hline Parameter & Exciter 1 & Exciter 2 & \\
\hline Poles & 4 & 4 & Unit \\
Maximum exciting force & 7 & 7 & Pole \\
Eccentric mass of the block of the exciter & 2.29 & 2.29 & $\mathrm{kN}$ \\
Rated power & 0.37 & 0.37 & $\mathrm{~kg}$ \\
Rated voltage & 380 & 380 & $\mathrm{~kW}$ \\
Rated current & 1.32 & 1.32 & $\mathrm{~V}$ \\
Stator resistance & 16.88 & 16.94 & $\mathrm{~A}$ \\
Rotor resistance & 12.63 & 12.63 & $\Omega$ \\
Stator inductance & 41.77 & 41.77 & $\mathrm{~m}$ \\
Rotor inductance & 40.10 & 44.09 & $\mathrm{mH}$ \\
\hline
\end{tabular}

TABLE 3: Main parameters of the vibration system.

\begin{tabular}{lccc}
\hline Parameter & Symbol & Value & Unit \\
\hline $\begin{array}{l}\text { Moment of inertia of the vibration } \\
\text { system }\end{array}$ & $J$ & 56.5 & $\mathrm{~kg} \times \mathrm{m}^{2}$ \\
Total mass of the vibration system & $M$ & 270.6 & $\mathrm{~kg}$ \\
$\begin{array}{l}\text { Stiffness coefficient in } x \text { direction } \\
\text { Stiffness coefficient in } y \text { direction }\end{array}$ & $k_{x}$ & 100 & $\mathrm{kN} / \mathrm{m}$ \\
Damping coefficient in $x$ direction & $c_{x}$ & 0.305 & $\mathrm{kN} / \mathrm{m}$ \\
$\begin{array}{l}\text { Damping coefficient in } y \text { direction } \\
\begin{array}{l}\text { Distance from a connecting point } \\
\text { between spring and frame to mass- }\end{array}\end{array}$ & $c_{y}$ & 0.282 & $\mathrm{~N} / \mathrm{mm}$ \\
center $o$ & $l_{s}$ & 0.525 & $\mathrm{~m}$ \\
\hline
\end{tabular}

To observe the stability of the vibration synchronization state and its phase difference distributions, the different distance $l$ should be considered along with the equivalent radius $l_{e}$ which is a constant for the system. Thus, $l=0.68 \mathrm{~m}$ and $l=1.36 \mathrm{~m}$ are taken for the experimental analysis at different rotational speeds of the exciters. A total of 24 groups of experiments are designed. The parameters of the experimental group are shown in Table 4.

The experimental process is limited to 100 seconds. To avoid the phenomenon of synchronous vibration
TABle 4: Experimental design when $l=0.68 \mathrm{~m}$ and $l=1.36 \mathrm{~m}$.

\begin{tabular}{lcccc}
\hline Item & \multicolumn{4}{c}{ Rotational speed ratio } \\
\hline$n=1$ & $1500: 1500$ & $1200: 1200$ & $900: 900$ & $500: 500$ \\
$n=2$ & $1500: 750$ & $1400: 700$ & $1200: 600$ & $1000: 500$ \\
$n=3$ & $1500: 500$ & $1410: 470$ & $1320: 440$ & $1200: 400$ \\
\hline
\end{tabular}

transmission, the high-speed exciter turns on firstly and the other exciter motor powers up after 3 seconds delay. If the difference between the maximum value or minimum value of the phase differences of two exciters cannot exceed 30 degrees more than 60 seconds during the experimental process, it will be regarded as achieving the vibration synchronization state, and the average phase differences of steady-state operation will be recorded; otherwise, it will not be considered as achieving the vibration synchronization state. The distributions of phase differences at steady state according to Table 4 are plotted in Figures 4-5, respectively.

In Figures 4 and 5, the left vertical coordinate represents the phase difference, which is statistic data of the steady-state value and represented by boxplot. The size of the box gives intervals of the upper quartile and lower quartile intuitively, in which the straight lines denote the mean value, maximum value, and minimum value, respectively. Also, the 


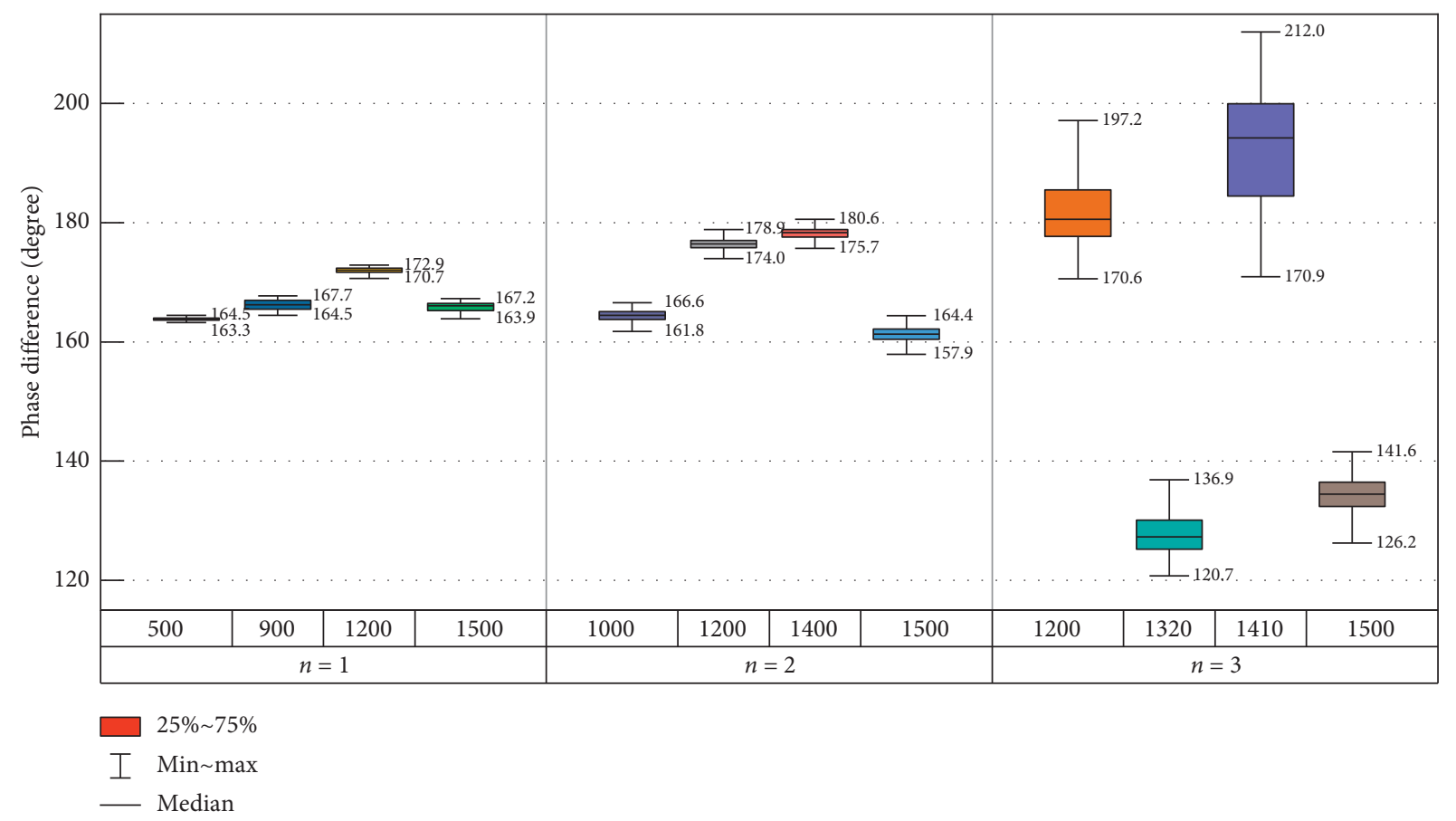

Figure 4: Distribution of phase differences of two exciters at $l=0.68 \mathrm{~m}$.

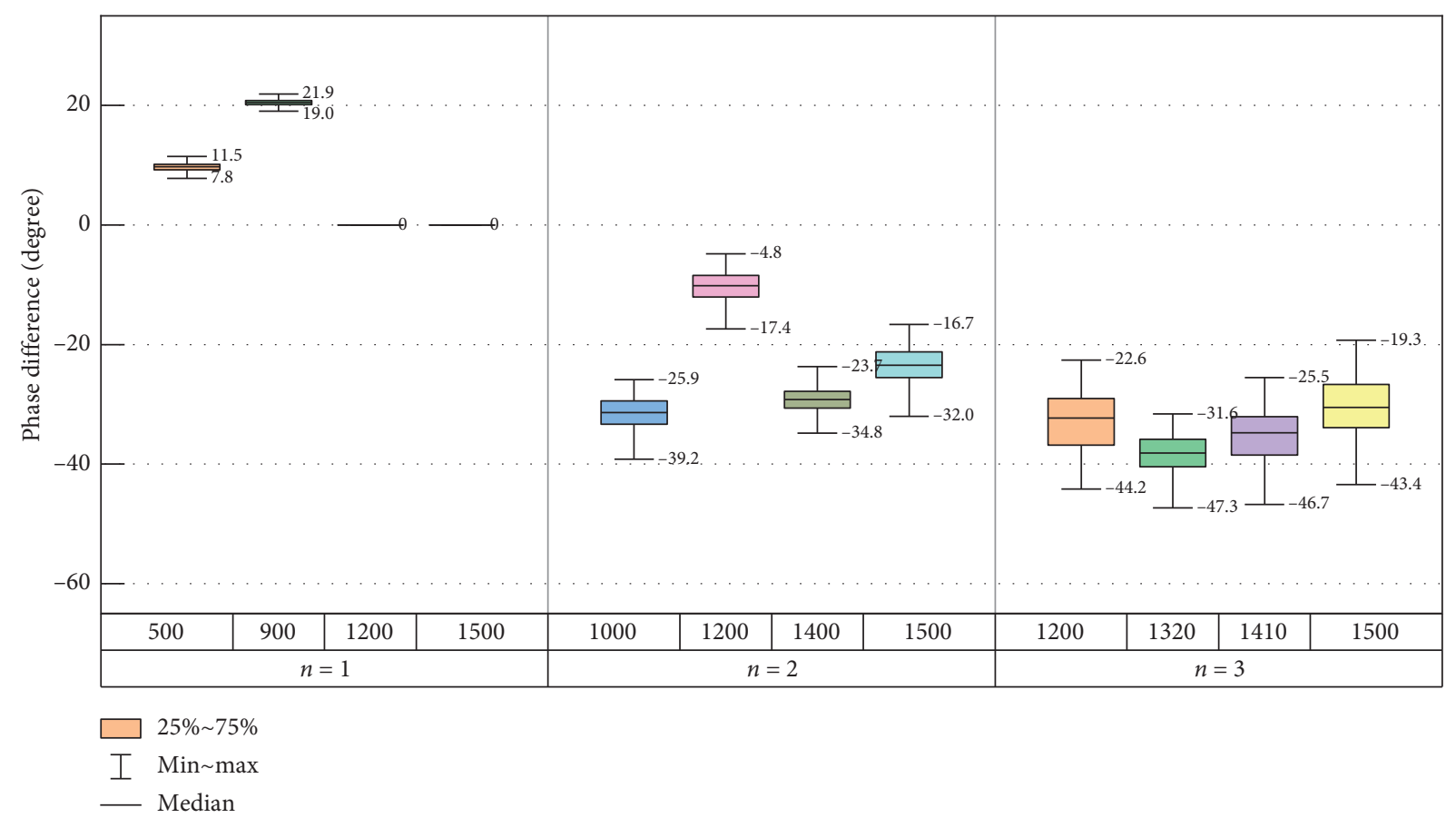

FIgURE 5: Distribution of phase differences of two exciters at $l=1.36 \mathrm{~m}$.

corresponding values are also labeled in the figures. The first row of the horizontal coordinate respects the multiple relations of the frequency of the two exciters, and the second row of the horizontal coordinate respects the rotational speed of exciter 1 .

Because the phenomena of vibration synchronization transmission appear quickly when $n=1, l=1.36 \mathrm{~m}$, $\omega_{2}=1200 \mathrm{r} / \mathrm{min}$, and $\omega_{2}=1500 \mathrm{r} / \mathrm{min}$, two groups of experimental data are missing in Figure 5. As shown in Figures 4 and 5, the different distribution intervals of phase difference are apparent, the distributions of the boxes in the same frequency and times-frequency experimental groups at $l=0.68 \mathrm{~m}$ and $l=1.36 \mathrm{~m}$, the former groups are stable near 180 degrees, the antiphase synchronization phenomenon appears, while the latter groups are stable near 0 degrees and the in-phase synchronization phenomenon comes out. It 
attributes to the existence of $\delta$ in equation (22) leads to the appearance of the bistable phase difference interval. At the same time, the length of boxes denote the vibration synchronization state at $n=1,2$ which is more steady than that when $n=3$. The time needed for the same frequency experimental group $(n=1)$ to achieve the vibration synchronization state is also shorter than other groups both at $l=0.68 \mathrm{~m}$ and $l=1.36 \mathrm{~m}$, which can be found in Figure 6 . The varying phase differences between the two exciters over time when $\omega_{1}=1200 \mathrm{r} / \mathrm{min}, n=1,2,3$, and $l=0.68 \mathrm{~m}$ and $\omega_{2}=500 \mathrm{r} / \mathrm{min}, n=1,2,3$, and $l=1.36 \mathrm{~m}$ are given in Figure 6 as examples, respectively. The rotational speed of the exciter over time is also plotted in Figure 7. Besides, the experiments also show the rotational speed of exciters has no prominent influence on the stability of the system.

From Figure 6, the synchronization state of the same frequency vibration is more comfortable to achieve and more stabilized than that of the times-frequency vibration synchronization state, which is coined with Figures 4 and 5 . The detailed quantitative analysis of the stability of the vibration state can also be found in the next section.

After collecting the pulse signals from the optical laser sensors to calculate the phase differences of the eccentric blocks of exciters, it can be defined as the differences between the angle of rotation $\varphi_{1}$ of exciter 1 to $\varphi_{2}$ of exciter 2 multiplied by the multiple relations of frequency $n$ when the phase of the low-speed exciter is viewed as the reference, i.e., $\alpha_{2}=0$. At the same time, for an easier way to illustrate the difference of phase differences at $l=0.68 \mathrm{~m}$ and $l=1.36 \mathrm{~m}$. The motion of the exciters is referred to a rectangular coordinate system and uses the mass center of blocks of lowspeed exciter passing the coordinate axis in a single circle as a reference. Thus, the increments of the rotational angle of the exciter and its instantaneous position can be examined over the same time. The corresponding real-time photos of the eccentric block of the exciters obtained by the high-speed camera are shown in Figures 8-13.

In Figure 6 and Figures 8-13, we can quickly note that the phase differences between the two homodromy exciters are stable around 180 degrees when they are located at a short distance; the antiphase synchronization appears. On the contrary, they are around 0 degrees at an in-phase synchronization state, which is coined with Figures 4 and 5 and further verifies the correctness of the theoretical deduction process. At the same time, we note that the distributions of phase differences of the exciters have not the same increase (or decreases) rule. This phenomenon is due to some external unconstant factors, especially the damping coefficients of the axes of the exciters, which are not constants when the exciters operate at different rotational directions and different rotational speeds. Meanwhile, for the vibrating system to achieve the vibration synchronization state, the exciters adjust themselves to the rotational phase continuously to establish the weak interaction. Thus, the boxplot does not have the same increase (or decrease) rule.

As mentioned in the previous section, on the one hand, times-frequency vibration machinery urgently needs to be developed from forced synchronization to vibration synchronization. On the other hand, the primary motion type is swing around the mass center of the system for the vibration system driven by two homodromy exciters under the same frequency powered supply when $r_{\psi}<\sqrt{2}$ as stated in [31], which have no obvious practical significance in engineering. However, the motion of purely swinging around the mass center cannot appear in the system of times-frequency vibration synchronization due to the inconsistent rotational speeds of the two exciters. To observe the trajectories of the system, a feeding material chamber is assembled upon the mass center of the system, and it enriches a part of the particle materials. Then, the influence of exciting force and trajectories of the materials can be visually obtained by observing the movement of material. We find that the particle materials in the chamber have not moved as a whole at $\omega_{1}=1200 \mathrm{r} / \mathrm{min}, \omega_{2}=1200 \mathrm{r} / \mathrm{min}$, and $l=0.68 \mathrm{~m}$ and $\omega_{1}=500 \mathrm{r} / \mathrm{min}, \omega_{2}=500 \mathrm{r} / \mathrm{min}$, and $l=1.36 \mathrm{~m}$. The former phenomenon is attributing to the system operates in a swing state. The latter is at the root of lacking enough exciting force when the two exciters rotate with a low speed. The other conditions denote that the particles are moving with the opposite direction against the exciters' rotation direction, i.e., the particles rotate as a whole in the clockwise direction when the two exciters rotate in the counterclockwise direction. Obviously, the forces acting on the particles come from the vibration of the shell; the exciting loads in the different positions at $n=1,2,3, \omega_{1}=1200 \mathrm{r} / \mathrm{min}$, and $l=0.68 \mathrm{~m}$ and $\omega_{2}=500 \mathrm{r} / \mathrm{min}_{\text {and }} l=1.36 \mathrm{~m}$ are shown in Figure 14. At the same time, the times-domain responses of them are also plotted, as shown in Figure 15 and Figure 16, where, the data of left figures, middle figures, and right figures are obtained from the leftmost, middle, and rightmost test point of the frame, denoted by (a), (b), and (c), respectively.

As shown in Figure 14, because of the existence of times-frequency loads, the force acting on the shell will not be regular circular, but other forms. Compared with the same frequency loads, the complex compound loads of the shell make the particle trajectories more complicated, which means that there are more mutual extrusion and friction in the particle materials in the chamber along with the process of particles rotating as a whole. It may have apparent practical significance in engineering. For example, for the vibration mill, the group motion of the particle materials will increase the actual contact friction surfaces and the crushing effects of the particles, which may significantly reduce the low-energy zone of milling particles and improve the milling efficiency. The related research is being pushed ahead, which may be discussed in future studies (Figure 16).

4.2. Quantitative Analysis. As mentioned above, the phenomenon of bistable phase interval in the system is caused by the angle $\delta$ when equation (21) is arranged in the form of equation (22). Specifically, $W_{\text {sic }}(i=1,2)$ is greater than or equal to 0 because of $\beta \in[0, \pi / 2]$. Thus, the positive or negative sign of $n^{2} W_{c 1 c}+W_{c 2 c}$ determines the stability interval of the phase difference of the vibration synchronization system, which mainly varies along with the parameters $r_{l}$ and $\beta$. The influence of $r_{l}$ and $\beta$ on $\delta$ is plotted in Figure 17. 


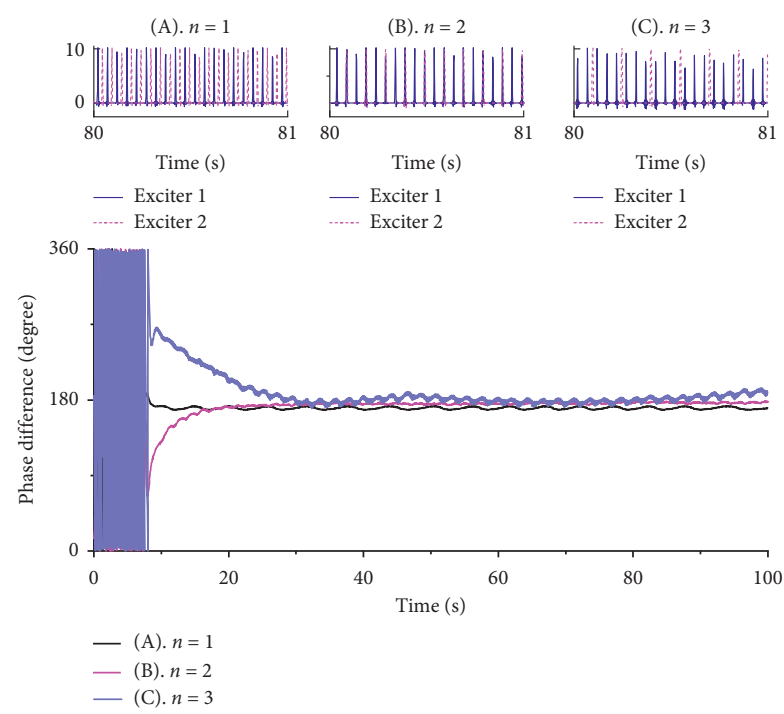

(a)

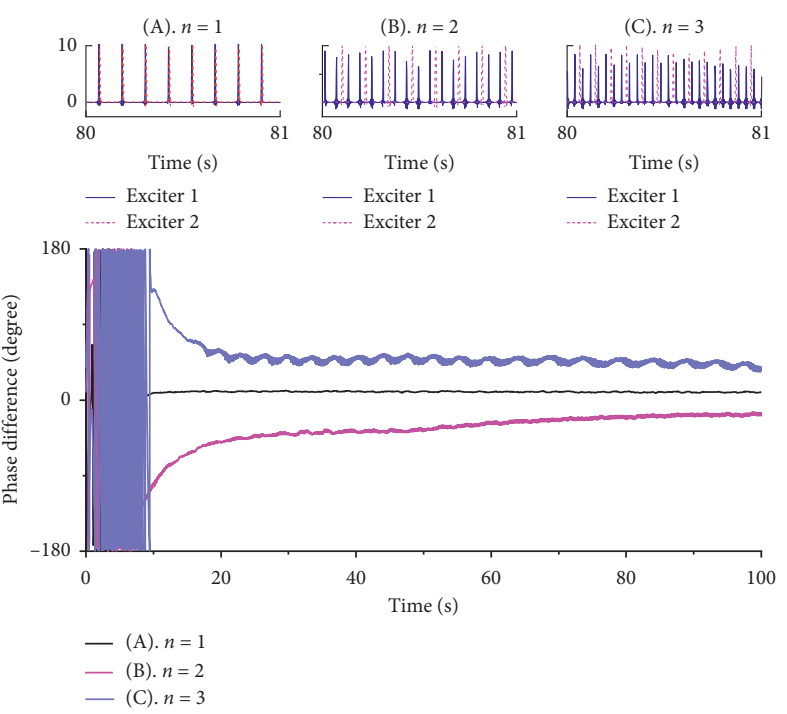

(b)

Figure 6: Phase differences between the two exciters. (a) $l=0.68 \mathrm{~m}$ and $\omega_{1}=1200 \mathrm{r} / \mathrm{min}$; (b) $l=1.36 \mathrm{~m}$ and $\omega_{2}=500 \mathrm{r} / \mathrm{min}$.

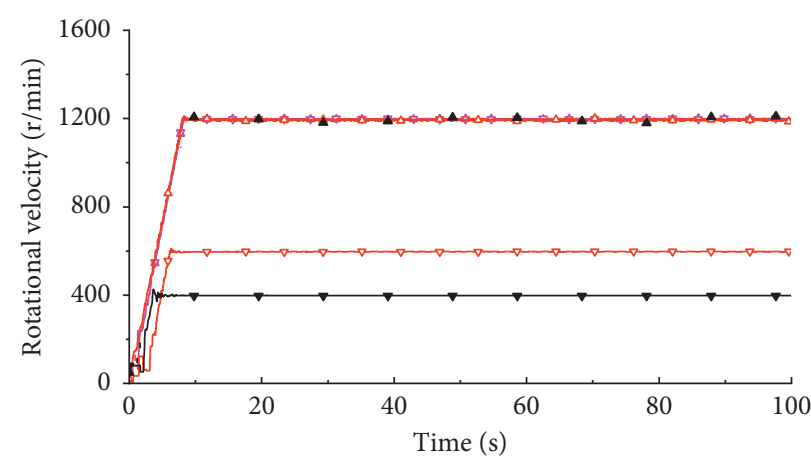
(A). $n=1$
$\triangle \omega_{1}=1200 \mathrm{r} / \mathrm{min}$
$\rightarrow \omega_{2}=1200 \mathrm{r} / \mathrm{min}$
(B). $n=2$
$\triangle-\omega_{1}=1200 \mathrm{r} / \mathrm{min}$
$\rightarrow \omega_{2}=600 \mathrm{r} / \mathrm{min}$
(C). $n=3$
$\simeq \omega_{1}=1200 \mathrm{r} / \mathrm{min}$
$\neg \omega_{2}=500 \mathrm{r} / \mathrm{min}$

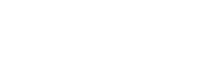

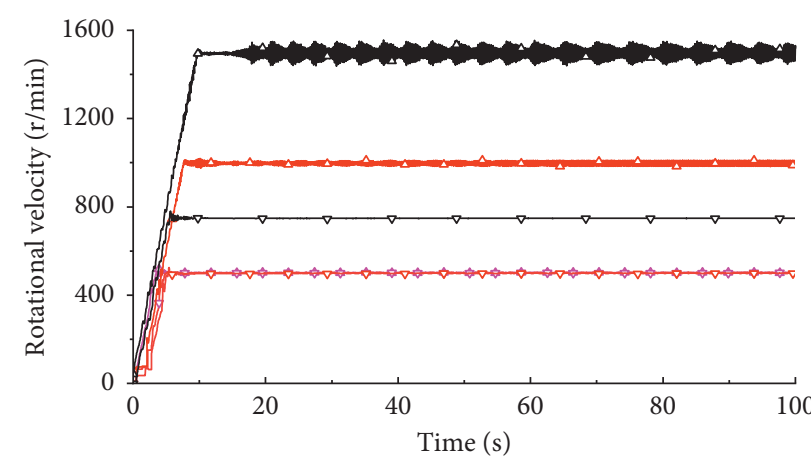

(A). $n=1$

$\triangle \omega_{1}=500 \mathrm{r} / \mathrm{min}$

(C). $n=3$

$\rightarrow \omega_{2}=500 \mathrm{r} / \mathrm{min}$

$\triangle \omega_{1}=500 \mathrm{r} / \mathrm{min}$

(B). $n=2$

$\triangle-\omega_{1}=500 \mathrm{r} / \mathrm{min}$

$\rightarrow \omega_{1}=1000 \mathrm{r} / \mathrm{min}$

(a)

(b)

Figure 7: Rotational velocity of the exciter over time. (a) $l=0.68 \mathrm{~m}$ and $\omega_{1}=1200 \mathrm{r} / \mathrm{min}$; (b) $l=1.36 \mathrm{~m}$ and $\omega_{2}=500 \mathrm{r} / \mathrm{min}$.

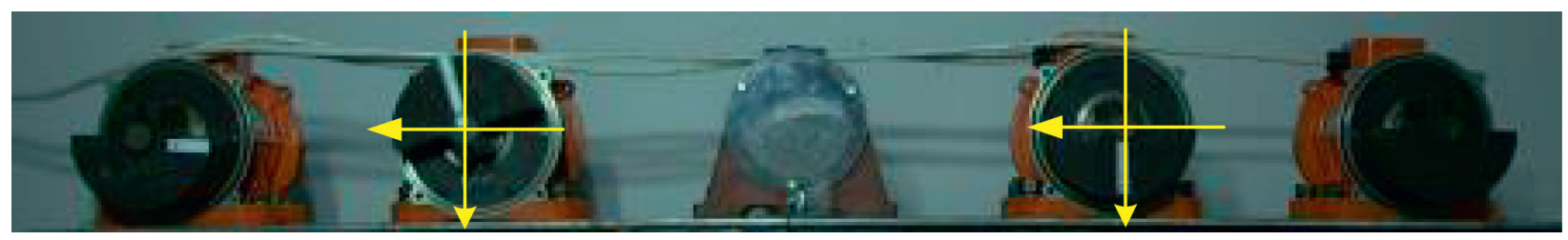

(a)

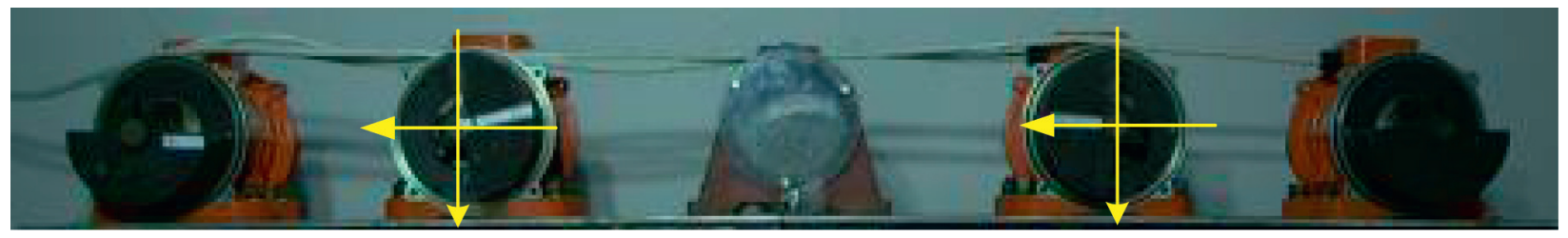

(b)

Figure 8: Continued. 


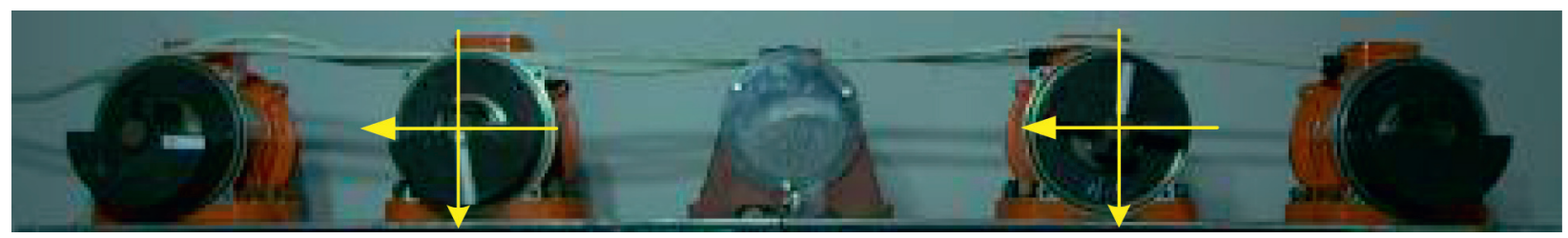

(c)

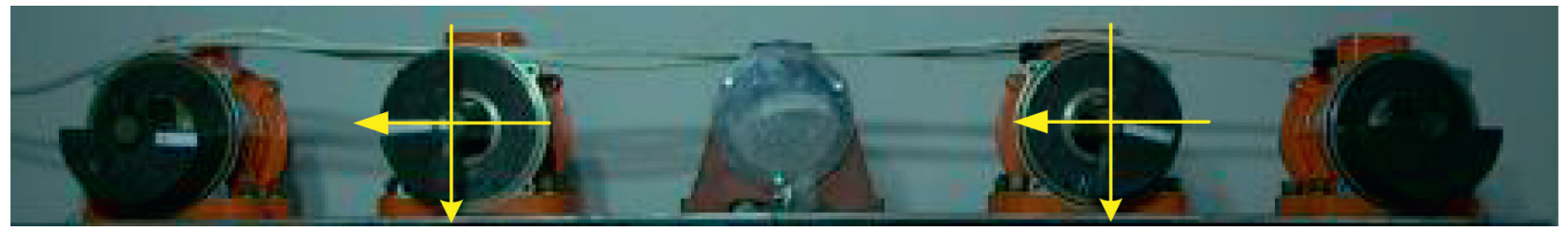

(d)

Figure 8: Instantaneous phase of the exciters when $l=0.68 \mathrm{~m}, n=1, \omega_{1}=1200 \mathrm{r} / \mathrm{min}$, and $\omega_{2}=1200 \mathrm{r} / \mathrm{min}:(\mathrm{a}) \varphi_{1}=170.3^{\circ}, \varphi_{2}=2.1^{\circ}$, and $\alpha=\varphi_{1}-\varphi_{2}=168.2^{\circ}$, (b) $\varphi_{1}=262.6^{\circ}, \varphi_{2}=93.2^{\circ}$, and $\alpha=\varphi_{1}-\varphi_{2}=169.4^{\circ}$, (c) $\varphi_{1}=355.2^{\circ}, \varphi_{2}=184.6^{\circ}$, and $\alpha=\varphi_{1}-\varphi_{2}=170.6^{\circ}$, and (d) $\varphi_{1}=86.1^{\circ}+360^{\circ}=449.1^{\circ}, \varphi_{2}=280.4^{\circ}$, and $\alpha=\varphi_{1}-\varphi_{2}=168.7^{\circ}$.

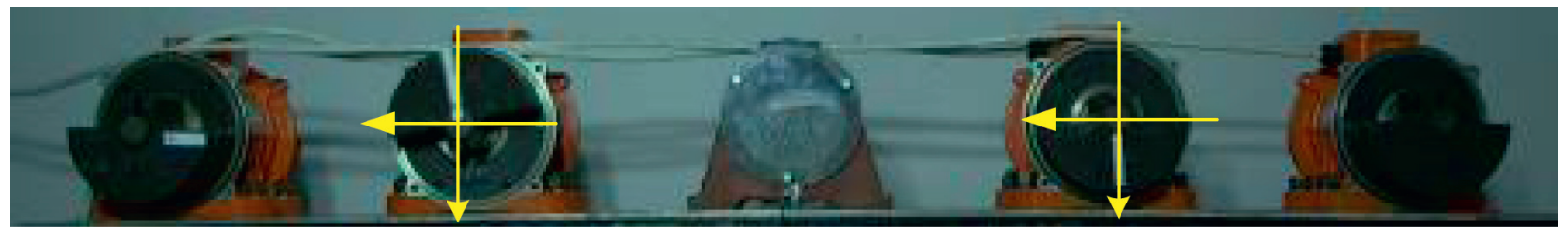

(a)

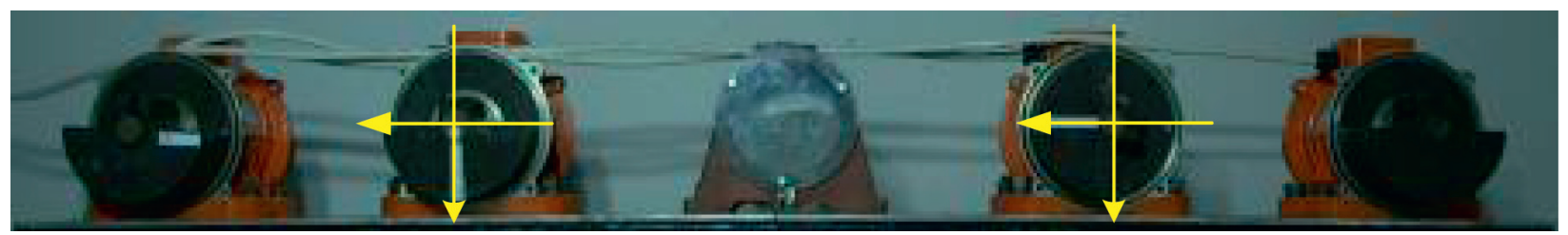

(b)

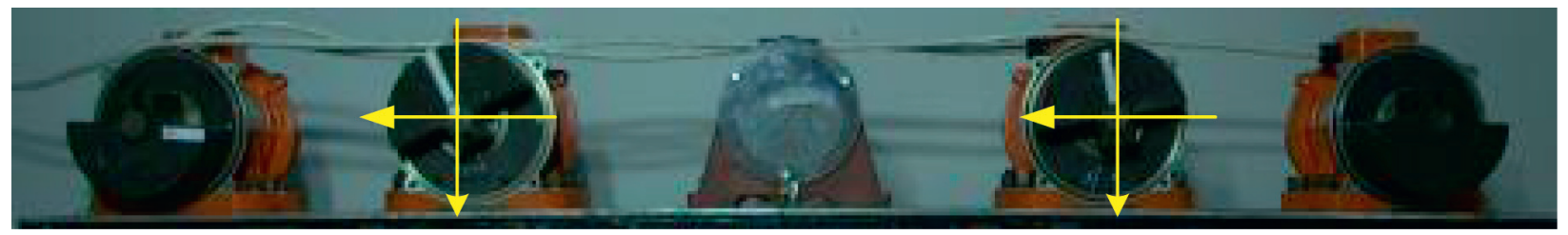

(c)

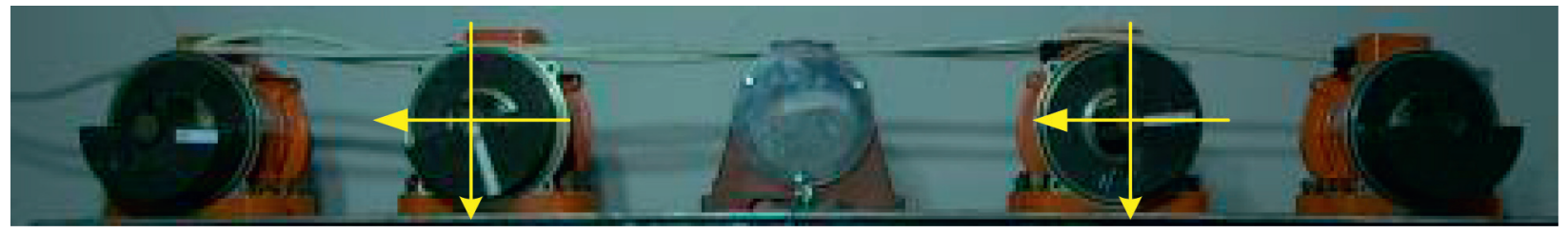

(d)

FIGURE 9: Instantaneous phase of the exciters when $l=0.68 \mathrm{~m}, n=1, \omega_{1}=1200 \mathrm{r} / \mathrm{min}$, and $\omega_{1}=1200 \mathrm{r} / \mathrm{min}:(\mathrm{a}) \varphi_{1}=168.3^{\circ}, \varphi_{2}=$ $-3.2^{\circ}$, and $\alpha=\varphi_{1}-2 \varphi_{2}=174.7^{\circ}$, (b) $\varphi_{1}=356.6^{\circ}, \varphi_{2}=90.2^{\circ}$, and $\alpha=\varphi_{1}-2 \varphi_{2}=176.2^{\circ}$, (c) $\varphi_{1}=156.2^{\circ}+360^{\circ}=516.2^{\circ}, \varphi_{2}=170.3^{\circ}$, and $\alpha=\varphi_{1}-2 \varphi_{2}=175.6^{\circ}$, and (d) $\varphi_{1}=345.4^{\circ}+360^{\circ}=705.4^{\circ}, \varphi_{2}=267.2^{\circ}$, and $\alpha=\varphi_{1}-2 \varphi_{2}=171.0^{\circ}$.

As shown in Figure 17, the influence of $r_{l}$ and $\beta$ on $\delta$ is more prominent. There is only one steady-state interval when $\beta>30^{\circ}$. The smaller $\beta$ denotes the more steady phase difference on $\delta$. It just can explain why many vibration machines require the composite mass center of the exciters, and the mass center of the vibration system is in a line to make the steady-state phase difference of the system approach to 0 or 180 , in which the synchronous state is more stable. For the experiments in the previous section, we have $r_{l}=1.56$ and $\beta=20.9^{\circ}$ when $l=0.68 \mathrm{~m}$ and $r_{l}=0.82$ and 


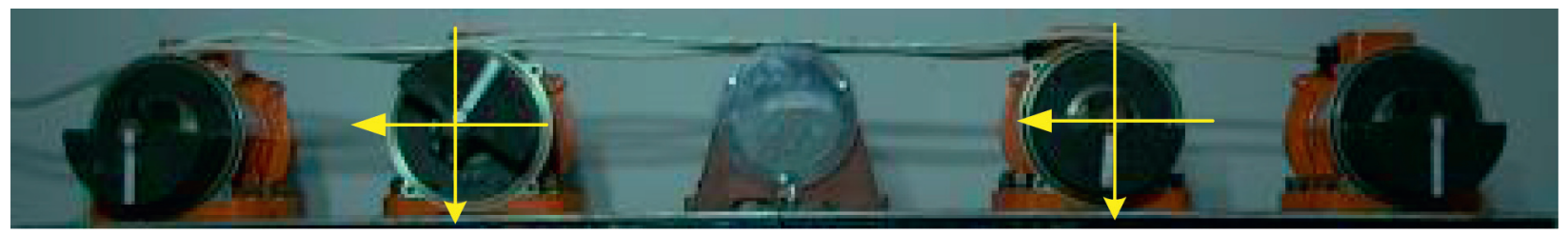

(a)

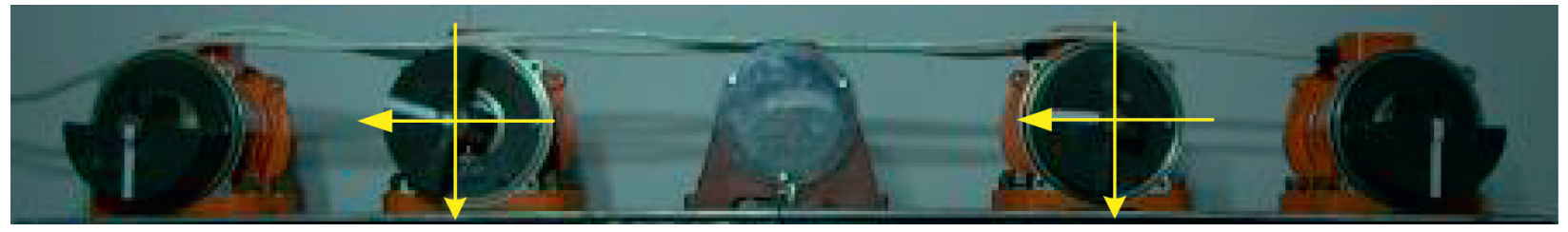

(b)

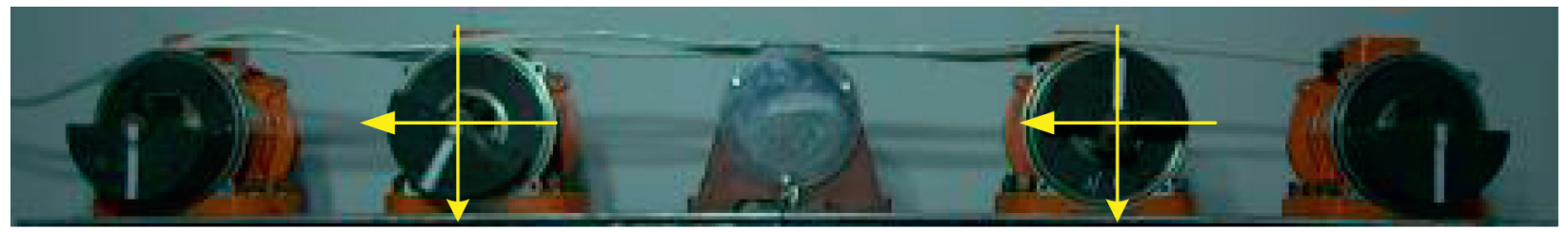

(c)

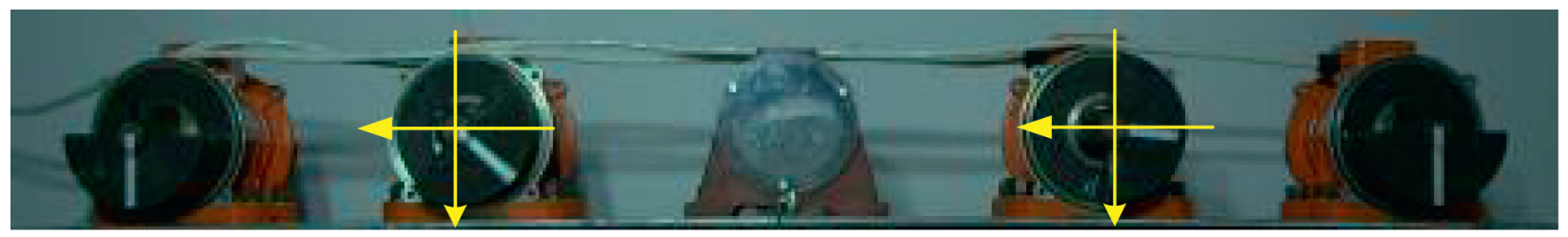

(d)

FIgURE 10: Instantaneous phase of the exciters when $l=0.68 \mathrm{~m}, n=3, \omega_{1}=1200 \mathrm{r} / \mathrm{min}$, and $\omega_{1}=400 \mathrm{r} / \mathrm{min}: \quad$ (a) $\varphi_{1}=213.3^{\circ}, \varphi_{2}=$ $6.5^{\circ}$, and $\alpha=\varphi_{1}-3 \varphi_{2}=193.8^{\circ}$, (b) $\varphi_{1}=107.7^{\circ}+360^{\circ}=467.7^{\circ}, \varphi_{2}=90.2^{\circ}$, and $\alpha=\varphi_{1}-3 \varphi_{2}=197.1^{\circ}$, (c) $\varphi_{1}=28.3^{\circ}+720^{\circ}=748.3^{\circ}, \varphi_{2}=$ $183.6^{\circ}$, and $\alpha=\varphi_{1}-3 \varphi_{2}=197.5^{\circ}$, and (d) $\varphi_{1}=310.2^{\circ}+720^{\circ}=1030.2^{\circ}, \varphi_{2}=278.8^{\circ}$, and $\alpha=\varphi_{1}-3 \varphi_{2}=193.8^{\circ}$.

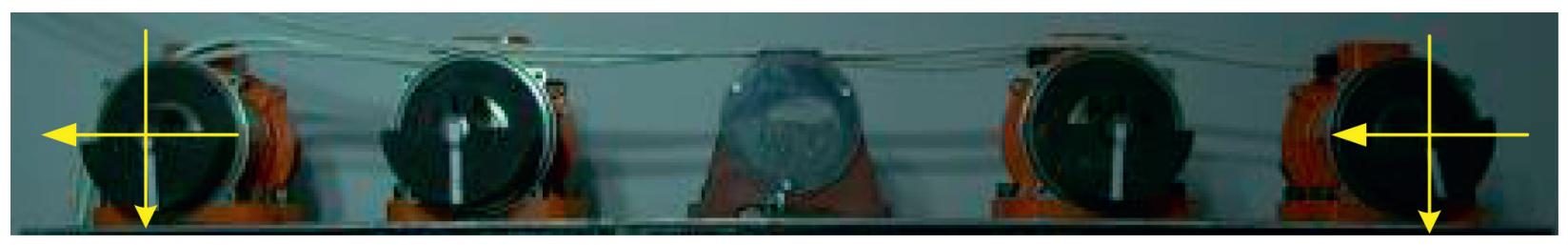

(a)

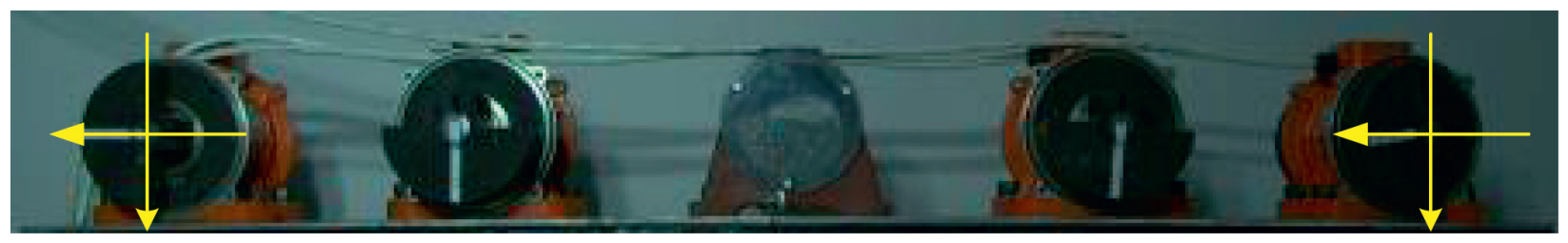

(b)

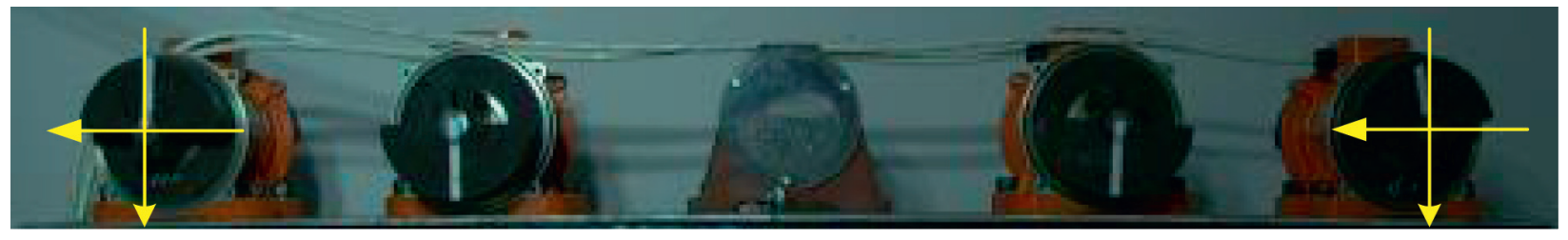

(c)

FIgUre 11: Continued. 


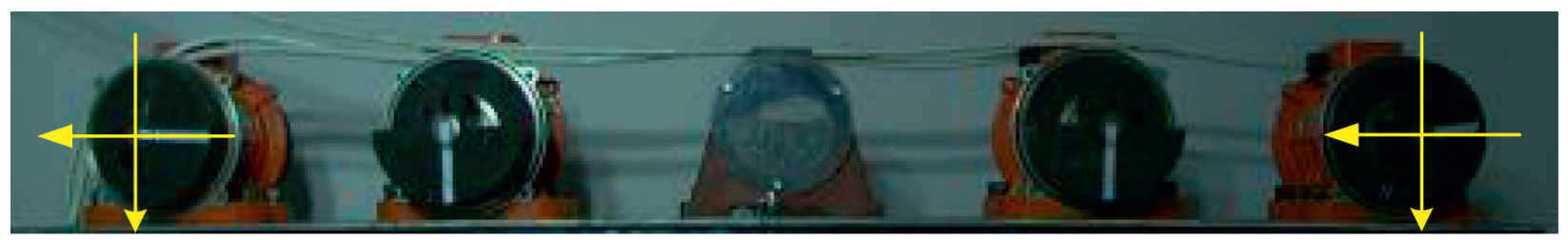

(d)

FIGURE 11: Instantaneous phase of the exciters at $l=1.3 \mathrm{~m}, n=1, \omega_{1}=500 \mathrm{r} / \mathrm{min}$, and $\omega_{1}=500 \mathrm{r} / \mathrm{min}:$ (a) $\varphi_{1}=-5.6^{\circ}, \varphi_{2}=-12.4^{\circ}$, and $\alpha=\varphi_{1}-\varphi_{2}=6.8^{\circ}$, (b) $\varphi_{1}=90.3^{\circ}, \varphi_{2}=82.5^{\circ}$, and $\alpha=\varphi_{1}-\varphi_{2}=7.8^{\circ}$, (c) $\varphi_{1}=184.1^{\circ}, \varphi_{2}=172.7^{\circ}$, and $\alpha=\varphi_{1}-\varphi_{2}=11.4^{\circ}$, and (d) $\varphi_{1}=270.3^{\circ}, \varphi_{2}=262.2^{\circ}$, and $\alpha=\varphi_{1}-\varphi_{2}=8.1^{\circ}$.

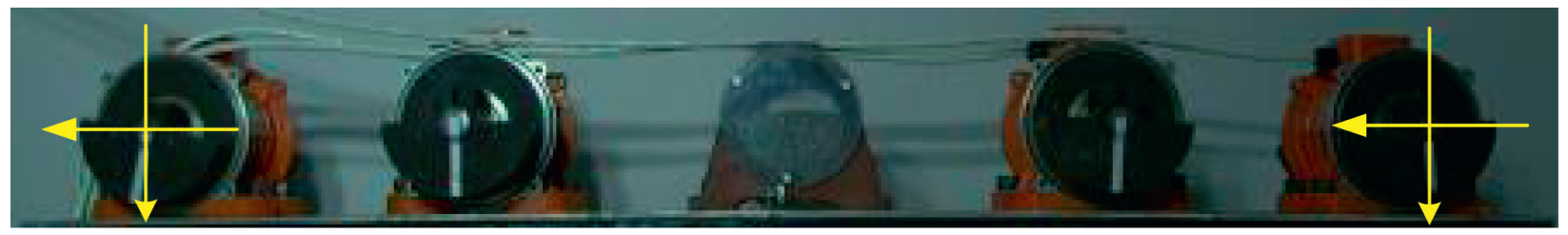

(a)

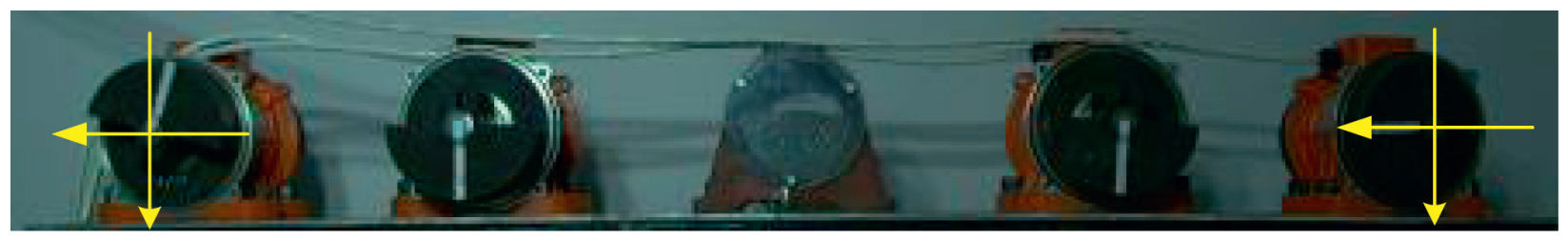

(b)

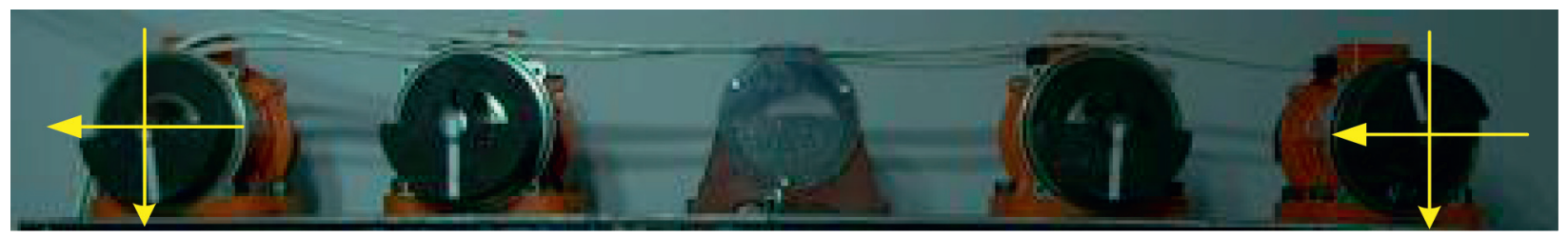

(c)

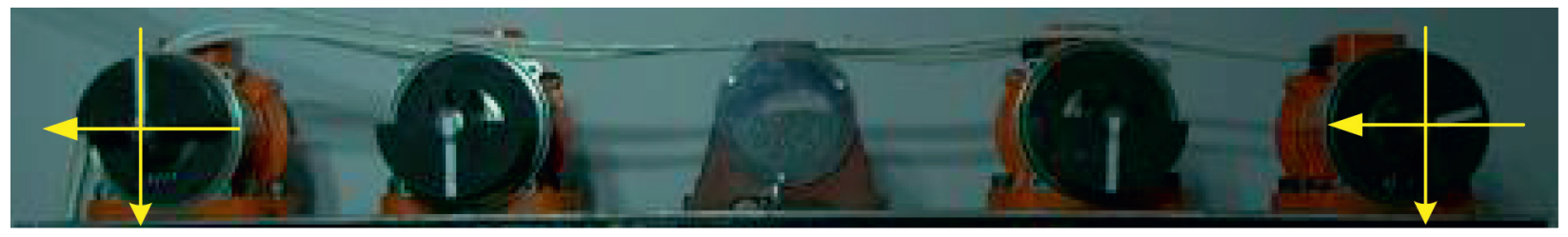

(d)

FiguRE 12: Instantaneous phase of the exciters at $l=1.3 \mathrm{~m}, n=2, \omega_{1}=1000 \mathrm{r} / \mathrm{min}$, and $\omega_{1}=500 \mathrm{r} / \mathrm{min}:(\mathrm{a}) \varphi_{1}=18.7^{\circ}, \varphi_{2}=-3.4^{\circ}$, and $\alpha=\varphi_{1}-2 \varphi_{2}=25.5^{\circ}$, (b) $\varphi_{1}=196.8^{\circ}, \varphi_{2}=85.4^{\circ}$, and $\alpha=\varphi_{1}-2 \varphi_{2}=26.0^{\circ}$, (c) $\varphi_{1}=350.2^{\circ}, \varphi_{2}=162.2^{\circ}$, and $\alpha=\varphi_{1}-2 \varphi_{2}=25.8^{\circ}$, and (d) $\varphi_{1}=179.3^{\circ}+360^{\circ}=539.3^{\circ}, \varphi_{2}=254.9^{\circ}$, and $\alpha=\varphi_{1}-2 \varphi_{2}=29.5^{\circ}$.

$\beta=10.8^{\circ}$ when $l=1.36 \mathrm{~m}$. Substituting the value of $\delta$ and the electrical parameters of exciters into equation (22), the phase difference between the simulation values and experimental results in Figures 4 and 5 are less than ten degrees, respectively, which can also be viewed in Figure 18. Also, the corresponding synchronization performance index $D$ calculated by equation (24) is listed in Figure 19, where the vertical coordinate is logarithmic.

As discussed in Section 1, to obtain a stable synchronization state for a system relying on the adjustment of oscillating objects due to their weak interaction. The more long distance between the two exciters means more weak interaction. This point can be coined in Figure 19 when $r_{l}<2$. Actually, $r_{l}$ is usually less than 2 in engineering. Thus, it is necessary to design the vibration system with a relatively shorter distance between the two exciters for achieving a robustness steady state, especially for the times-frequency vibration system. Compared with two and three times-frequency vibration synchronization system, the same frequency vibration system has more obvious stability. Also, two times-frequency systems is also more stable than three times-frequency synchronous systems, as shown in Figure 19. At the same time, the inflection point of bistable intervals shows poor stability, especially for the system with $\beta=0$. 


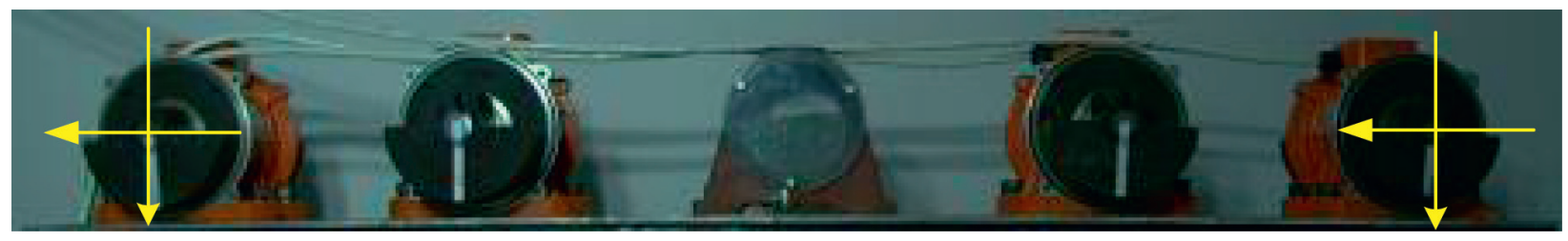

(a)

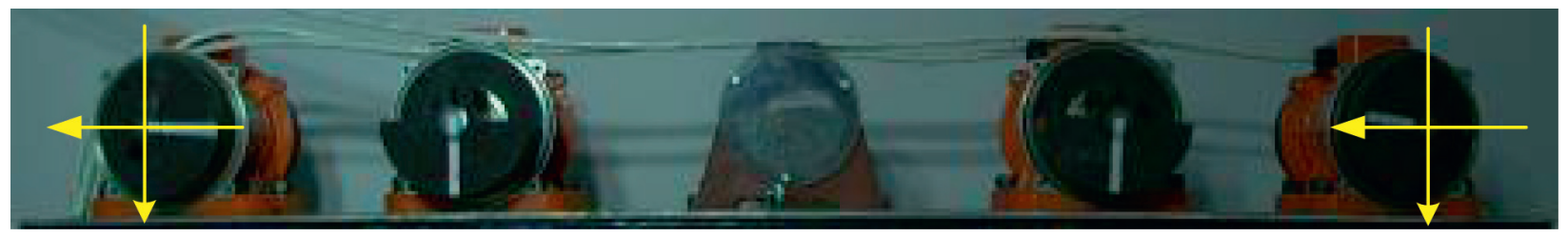

(b)

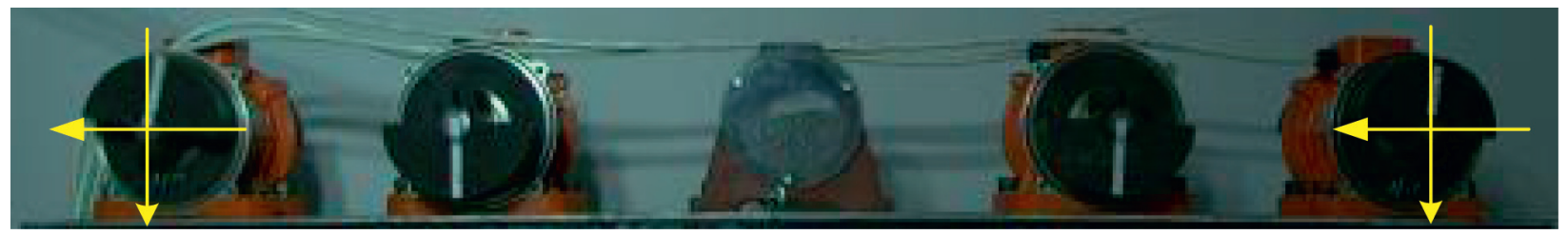

(c)

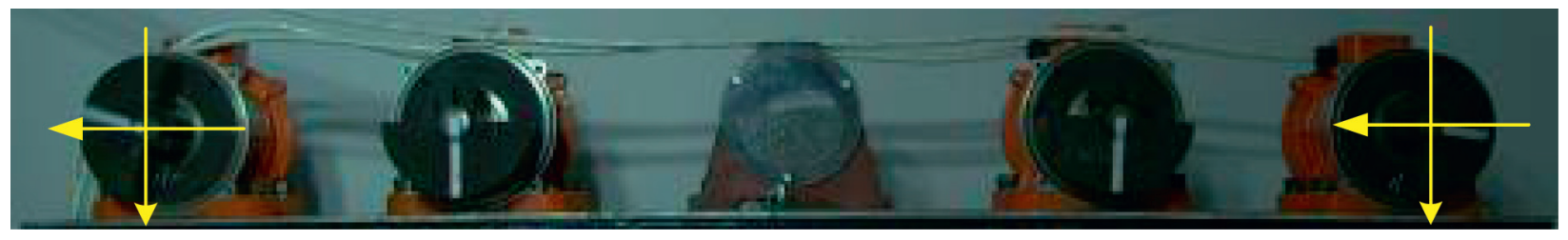

(d)

Figure 13: Instantaneous phase of the exciters at $l=1.3 \mathrm{~m}, n=3, \omega_{1}=1500 \mathrm{r} / \mathrm{min}$, and $\omega_{1}=500 \mathrm{r} / \mathrm{min}:(\mathrm{a}) \varphi_{1}=-7.2^{\circ}, \varphi_{2}=5.4^{\circ}$, and $\alpha=\varphi_{1}-3 \varphi_{2}=-23.4^{\circ}$, (b) $\varphi_{1}=272.8^{\circ}, \varphi_{2}=97.6^{\circ}$, and $\alpha=\varphi_{1}-3 \varphi_{2}=-20.0^{\circ}$, (c) $\varphi_{1}=189.0^{\circ}+360^{\circ}=549^{\circ}, \varphi_{2}=188.1^{\circ}$, and $\alpha=\varphi_{1}-3 \varphi_{2}=-15.3^{\circ}$, and (d) $\varphi_{1}=105.3^{\circ}+720^{\circ}=825.3^{\circ}, \varphi_{2}=282.1^{\circ}$, and $\alpha=\varphi_{1}-3 \varphi_{2}=-21.0^{\circ}$.

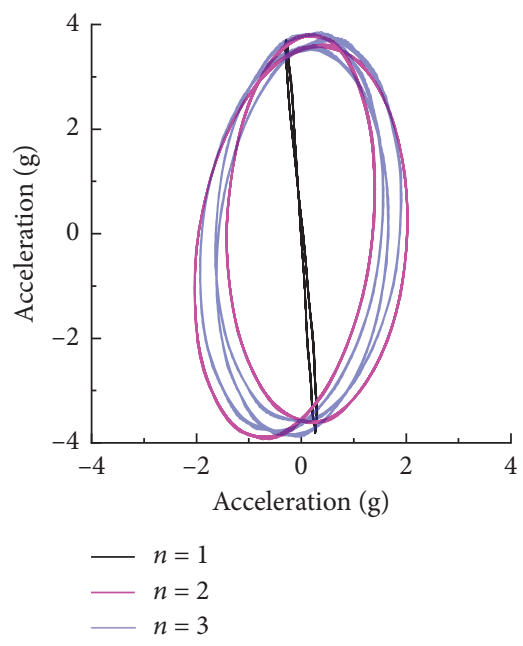

(A)

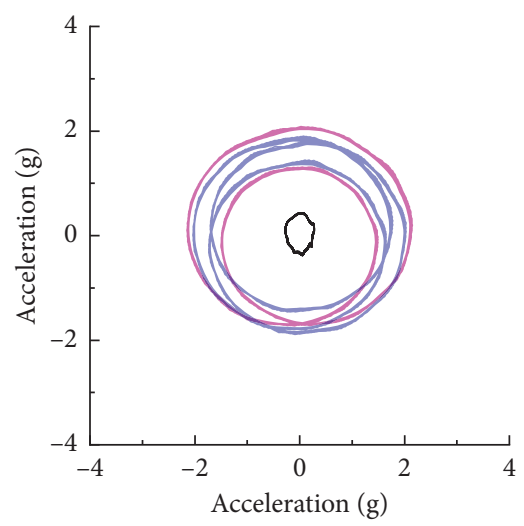

$\begin{aligned} n & =1 \\ & n=2 \\ -n & =3\end{aligned}$

(B)

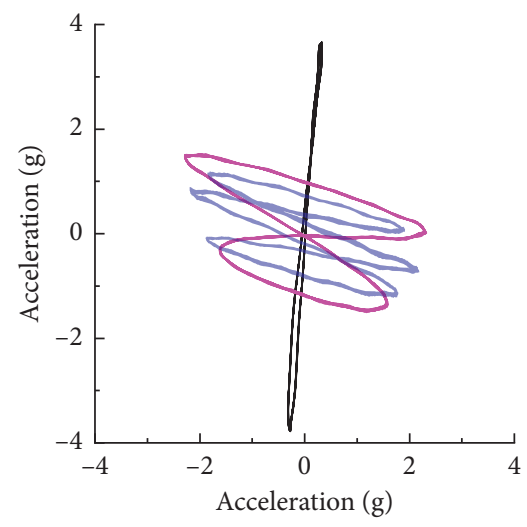

$\begin{aligned} n & =1 \\ -n & =2 \\ -n & =3\end{aligned}$

(C)

(a)

Figure 14: Continued. 


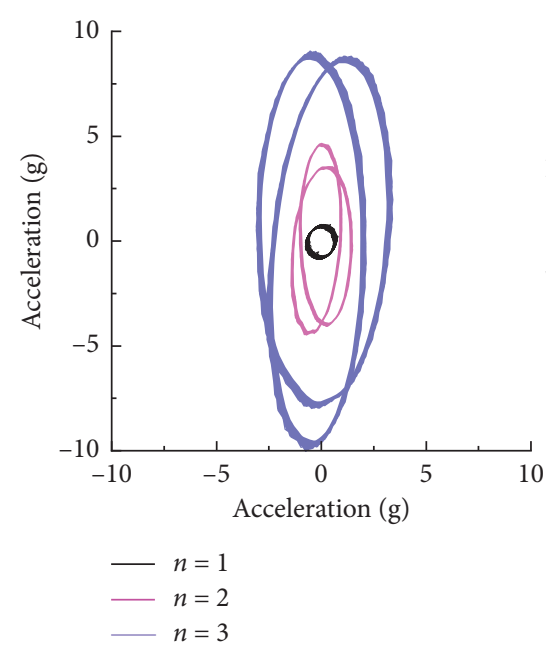

(A)

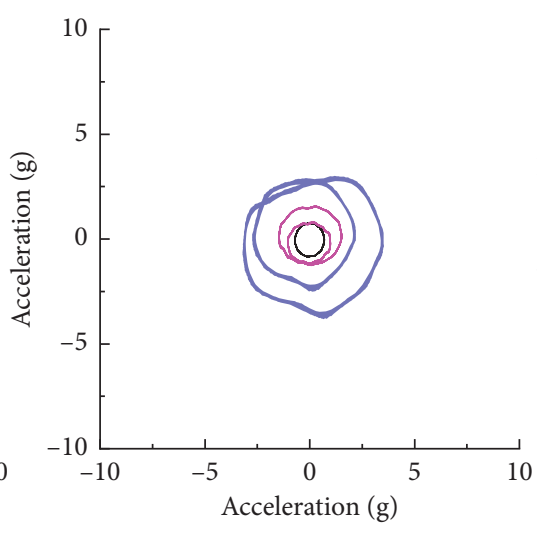

$\begin{aligned} n & =1 \\ -n & =2 \\ n & =3\end{aligned}$

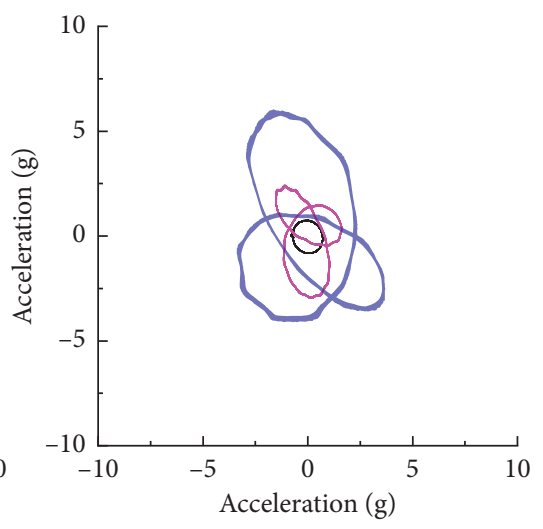

$-n=1$

- $n=2$

(B)

(C)

(b)

Figure 14: Exciting loads of the times-frequency system in different positions.

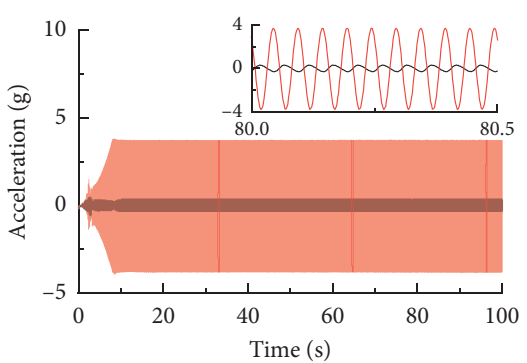

$-x$

(A)

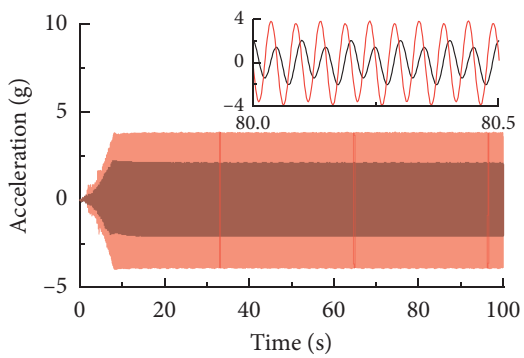

(A)
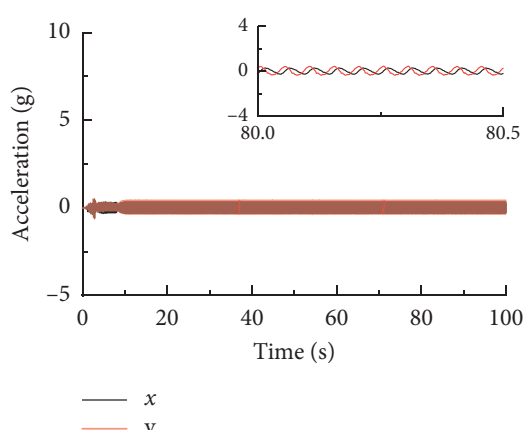

(B)

(a)

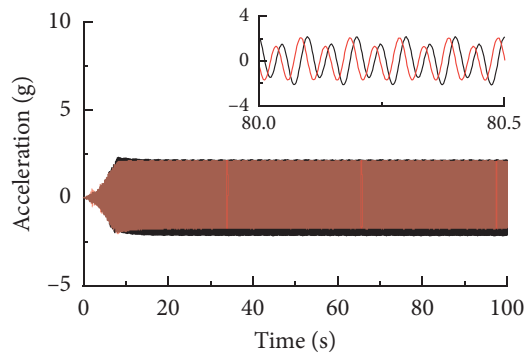

(B)
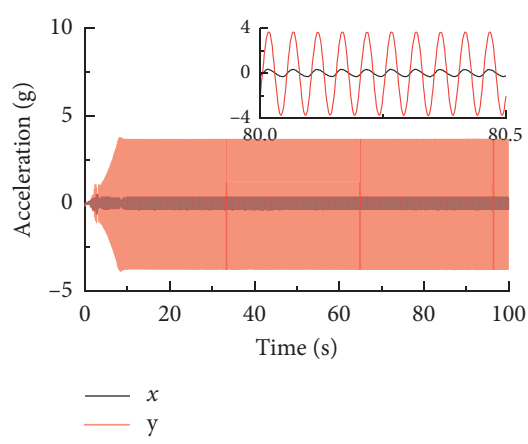

(C)

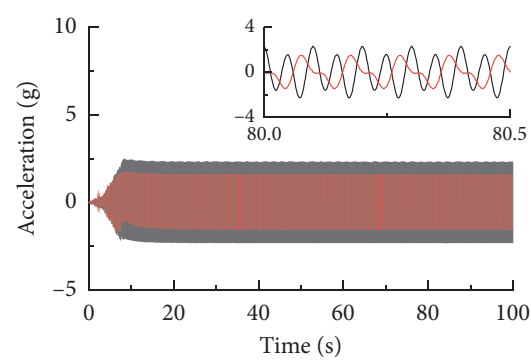

$x$
$-y$

(b)

Figure 15: Continued. 


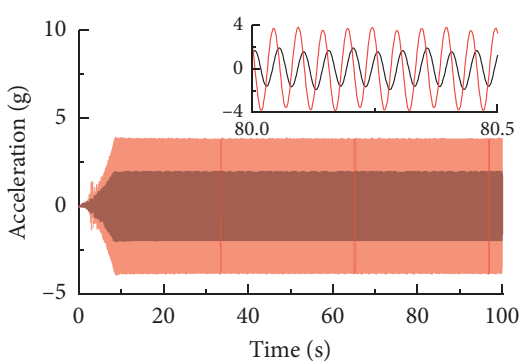

$x$
$-y$

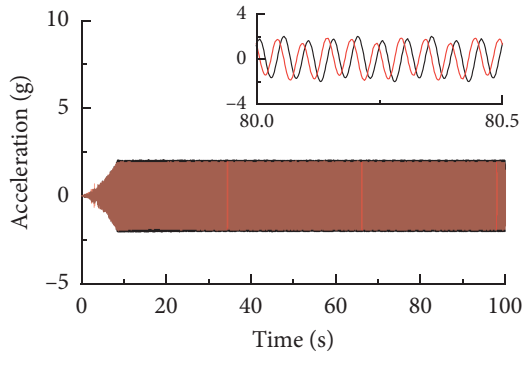

(B)

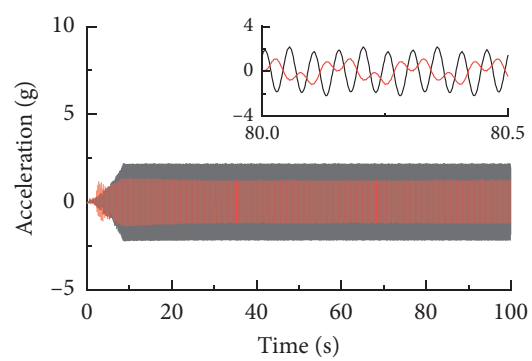

$x$
$-y$

(C)

(c)

FIGURE 15: Time-domain responses in different positions at $l=0.68 \mathrm{~m}$. (a) $n=1, \omega_{1}=1200 \mathrm{r} / \mathrm{min}$, and $\omega_{2}=1200 \mathrm{r} / \mathrm{min}$; (b) $n=2, \omega_{1}=1200 \mathrm{r} /$ $\mathrm{min}$, and $\omega_{2}=600 \mathrm{r} / \mathrm{min}$; (c) $n=3, \omega_{1}=1200 \mathrm{r} / \mathrm{min}$, and $\omega_{2}=400 \mathrm{r} / \mathrm{min}$.
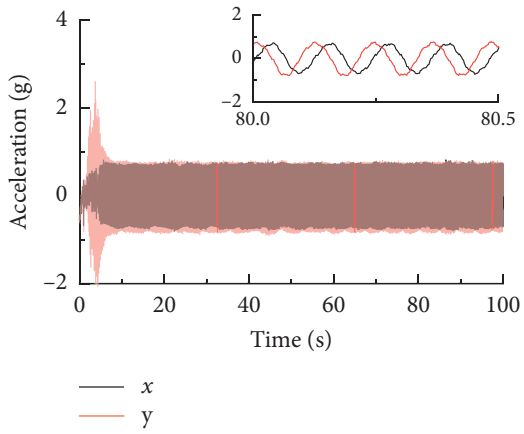

(A)

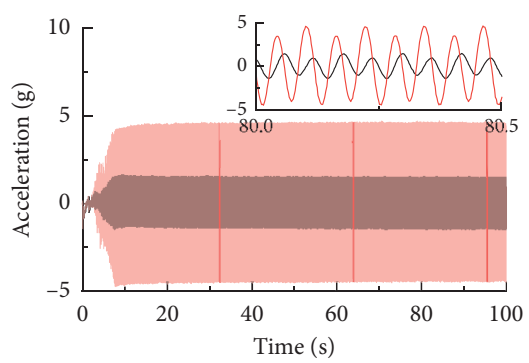

(A)
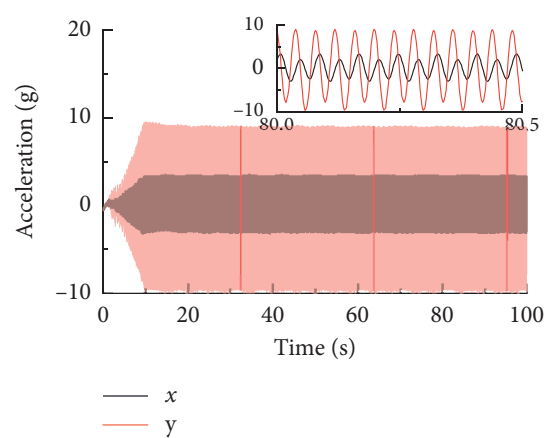

(A)

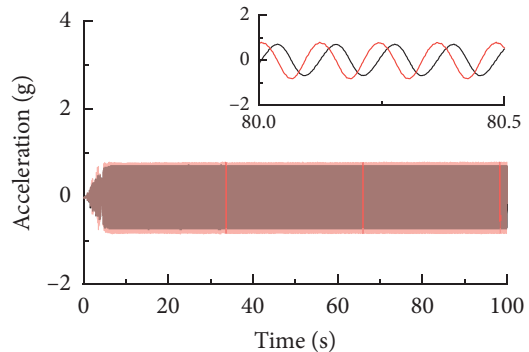

$-\mathrm{x}$

(B)

(a)

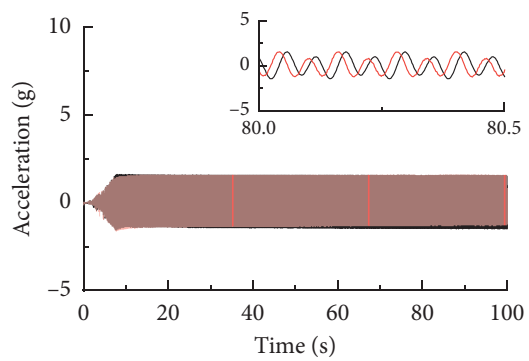

(B)

(b)

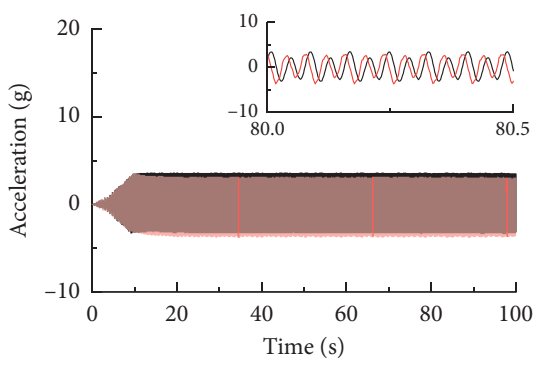

(B)

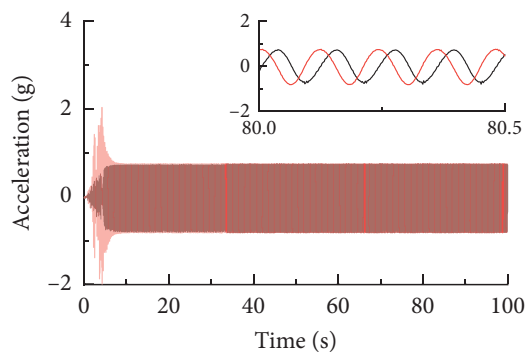

- $\begin{array}{r}x \\ \mathrm{y}\end{array}$

(C)

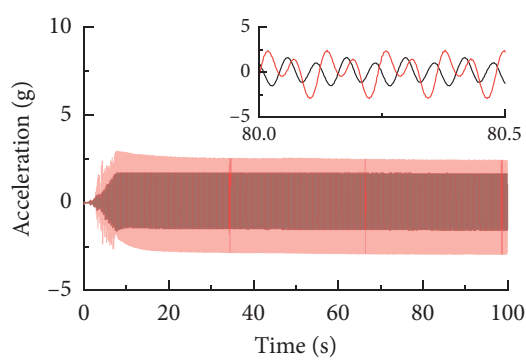

(C)

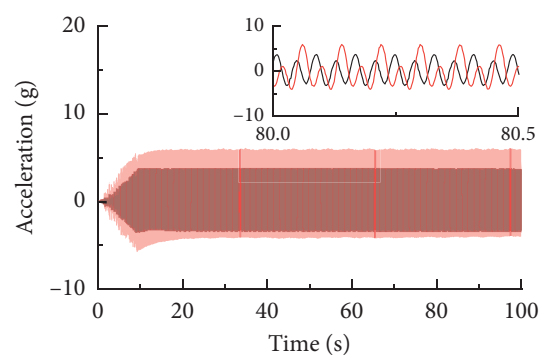

(C)

(c)

FIGURE 16: Time-domain responses in different positions at $l=1.36 \mathrm{~m}$. (a) $n=1, \omega_{1}=500 \mathrm{r} / \mathrm{min}$, and $\omega_{2}=500 \mathrm{r} / \mathrm{min}$; (b) $n=2, \omega_{1}=1000 \mathrm{r} / \mathrm{min}$, and $\omega_{2}=500 \mathrm{r} / \mathrm{min}$; (c) $n=3, \omega_{1}=1500 \mathrm{r} / \mathrm{min}$, and $\omega_{2}=500 \mathrm{r} / \mathrm{min}$. 


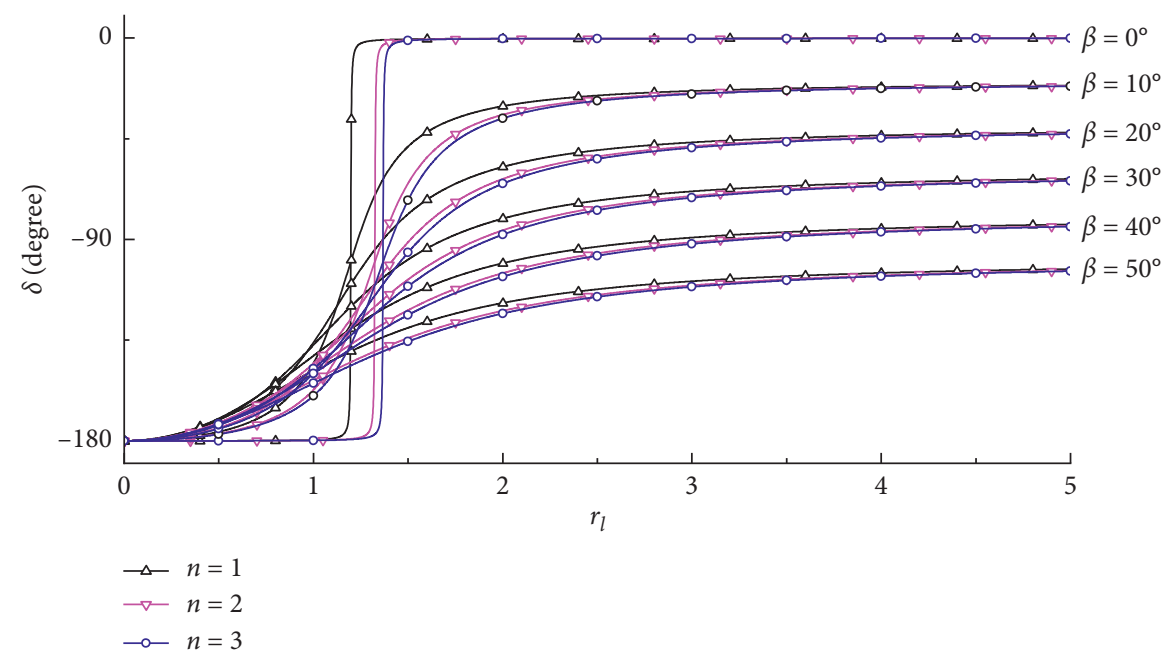

FIgURE 17: Influence of $r_{l}$ and $\beta$ on $\delta$ at $\omega_{2}=500 \mathrm{r} / \mathrm{min}$.

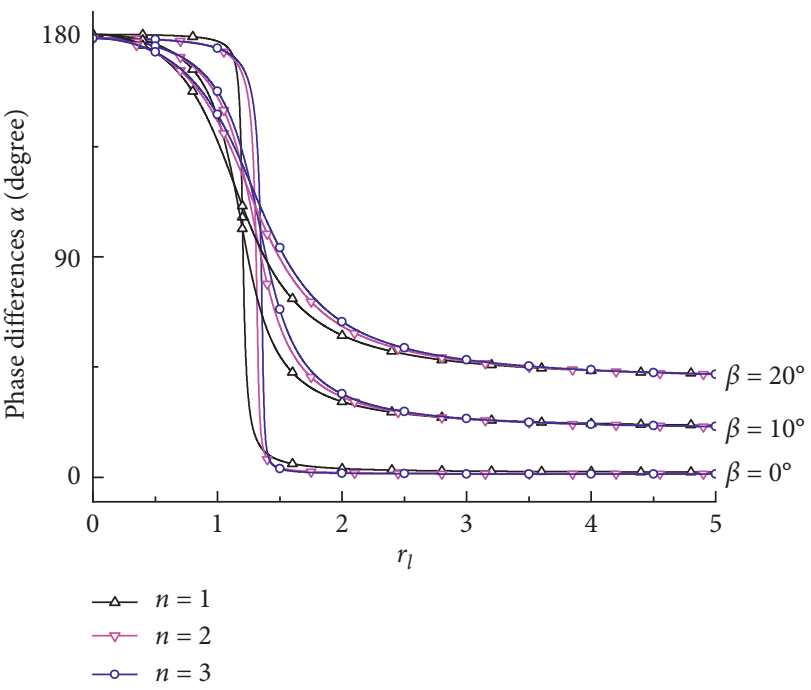

Figure 18: Phase difference of $\alpha$.

Because of the limiting minimum natural frequency of the supporting springs and the energy consumption reduction, when designing times-frequency synchronous machinery in engineering, it is better to choose smaller $\beta \approx 0, r_{l}<0.5$ and a higher rotational speed of the exciters to make the system realize the in-phase synchronization in a super-resonance state. As for the antiphase synchronization system, $r_{l}$ is greater than 2 is the right choice, which is can also be found in Figures 17-19.

Besides, for the bistable phase interval approaching to 0 or 180 degrees, the stability criterion (equation (31)) under angular velocity perturbation can be satisfied even if $\tau_{1}$ and $\tau_{2}$ are very small when using some other torque-slip models of the mechanical characteristic for the asynchronous motor. Similarly, the stability criterion of the phase difference perturbation system under phase difference perturbation can also be proved by substituting the parameter $\alpha$ and some other required parameters. Therefore, under the condition that the stability of the system can be satisfied, the

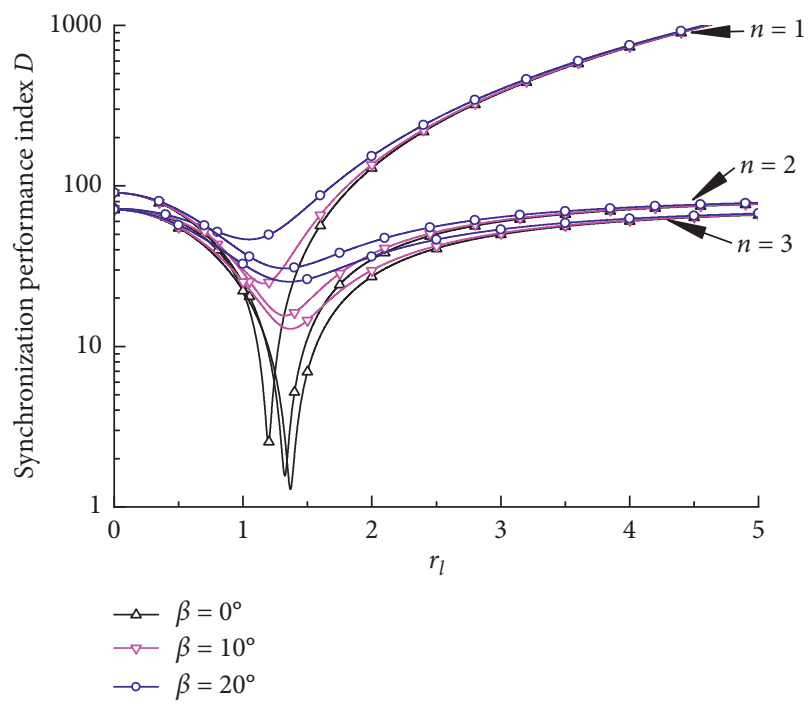

Figure 19: Synchronization performance index $D$.

synchronization performance index of the system becomes more significant in engineering.

\section{Conclusions}

The mechanical model of a times-frequency vibration system driven by two homodromy exciters is established to investigate whether the phenomenon of bistable phase difference intervals exists in the system. Based on small parameter perturbation methods and nonlinear system theory, the system has been studied with detailed theoretical and experimental research. Some useful conclusions can be given as follows.

Firstly, by introducing a group of average angular velocity perturbation parameter $\varepsilon_{0}$ and two groups of phasedifference perturbation parameters $\varepsilon_{1}$ and $\varepsilon_{2}$, the frequency capture conditions for times-frequency vibration synchronization of the system are derived. The phenomenon of bistable intervals of the phase difference of the system is 
revealed. When $r_{l}$ is smaller, we have $n^{2} W_{c 1 c}+W_{c 2 c} \geq 0$; the system is stable around 180 degrees and the antiphase synchronization phenomenon appears; on the contrary, while the system is stable near 0 degrees, the in-phase synchronization phenomenon comes out. Then, the frequency capture equation of the times-frequency synchronization system is linearized near the stable value of the phase difference $\alpha_{0}$ in the steady state and equivalent to a uniform perturbation system. Finally, the stability of the system under angular velocity perturbation and phase-difference perturbation are proved.

According to the mechanical model, a vibration system is established for verifying the phenomenon of bistable phase-difference intervals. The distance between the two exciters $l=0.68 \mathrm{~m}$ and $l=1.36 \mathrm{~m}$ are taken for the experimental analysis at different rotational speeds of the exciters. A material chamber is set upon the mass center of the vibration system to observe its trajectories. Our results show that the system mainly operates in a swing state around the mass center in the synchronous state of the same frequency with a smaller distance between the exciters due to the phase difference near $180^{\circ}$, and its engineering significance is low. For the times-frequency vibration synchronization, because of the existence of multiple rotational speeds of exciters, no matter what the value of $r_{l}$, the system presents a more complex compound motion trajectories.

Then, the quantitative analysis for the phenomenon of bistable phase-difference interval and the synchronization performance index is carried out. The same frequency vibration system has more obvious stability than the timesfrequency vibration synchronization system. The results show that it is better to choose smaller $\beta \approx 0, r_{l}<0.5$ and higher rotational speeds of the exciters in a super-resonance state when designing the time-frequency synchronous machinery. The complex compound motion trajectories of the times-frequency vibration system can be used to reduce the low-energy region of vibration mills and develop the chaotic mixing equipment.

Compared with the existing results, some innovations of this work can be summarized. Aiming at the familiar problem of vibration synchronization system with two exciters, times-frequency vibration synchronization theory of two exciters is developed; the phenomenon of bistable phase-difference intervals is given out. At the same time, the in-phase and antiphase synchronization phenomena for the times-frequency vibrating system are proved by experiments and its corresponding application is also investigated and discussed (Table 1).

\section{Nomenclature}

$o$ : $\quad$ Mass center of the whole vibration system

oxy: Fixed coordinate system

$o^{\prime} x^{\prime} y^{\prime}$ : Moving coordinate system

$m$ : Mass of the rigid frame

$m_{r}$ : Mass of the eccentric block

$m_{i}$ : $\quad$ Mass of the eccentric block of the exciter $i(i=1,2)$
$M: \quad$ Total mass of the vibration system

$k_{x}, k_{y}$ : Stiffness of the system in $x, y$ direction

$k_{x i}, k_{y i}$ : Stiffness of the spring $i(i=1,2)$ in $x, y$ direction

$c_{x}, c_{y}$ : Damping of the system in $x, y$ direction

$c_{x i}, c_{y i}$ : Damping of the spring $i(i=1,2)$ in $x, y$ direction

$c_{1}, c_{2}$ : Damping coefficient of the axes of the exciter $i(i=1,2)$

$r_{i}$ : $\quad$ Eccentric radius of the eccentric block of the exciter $i(i=1,2)$

$\dot{\varphi}_{i}: \quad$ Instantaneous angular velocity and the angle of rotation relative to the starting point of the exciter $i(i=1,2)$

$l_{0}$ : $\quad$ Distance from the composite mass center of the two exciters to the mass center of the system

$l$ : $\quad$ Distance from the axis of exciter 1 to the axis of exciter 2

$l_{i}$ : $\quad$ Distance from the axis of exciter $i(i=1,2)$ to the mass center $o$ of the system

$l_{s i}: \quad$ Distance from connecting point between spring $i(i=1,2)$ and frame to point $o$

$\beta_{0}$ : Angle between the connection line $l_{0}$ and the horizontal direction

$\beta_{i}$ : $\quad$ Angle between the connection line $l_{i}(i=1,2)$ and the horizontal direction

$\theta_{i}$ : Angle between the connection line $l_{s i}(i=1,2)$ and the horizontal direction

$J_{p}: \quad$ Moment of inertia of the rigid frame of the system about $o$

$J_{i}$ : $\quad$ Moment of inertia of the eccentric mass of exciter $i(i=1,2)$ to its mass center

$J$ : $\quad$ Moment of inertia of the vibration system about $o$

$T: \quad$ Kinetic energy of the system

$V: \quad$ Potential energy of the system

$D_{0}$ : $\quad$ Energy dissipate function of the system

$L_{e i}$ : Output torque of the inducing motor of exciter $i(i=1,2)$

$\alpha_{i}$ : $\quad$ Steady-state phase difference of exciter $i(i=1,2)$ in a single cycle

$n$ : Multiple relationships of rotational speed between the two exciters

$T_{0}$ : $\quad$ Single cycle of the high-speed exciter

$\omega_{n i}: \quad$ Natural frequency of the vibrating system in $i(i=x, y, \psi)$ direction

$\xi_{n x}$ : Critical damping ratios of the vibrating system in $i(i=x, y, \psi)$ direction

$\varepsilon_{0}$ : $\quad$ Small perturbation parameter about average speed $\dot{\varphi}$

$\varepsilon_{i}$ : $\quad$ Small phase perturbation parameter of exciter $i(i=1,2)$

$\bar{\varepsilon}_{i}: \quad$ Average values of $\varepsilon_{i}(i=0,1,2)$ in a single cycle

$\dot{\bar{\varepsilon}}_{i}$ : $\quad$ Average values of $\dot{\varepsilon}_{i}(i=0,1,2)$ in a single cycle

$k_{e 0 i}$ : Stiffness coefficients of the angular velocity of the motor of exciter $i(i=1,2)$ at a certain speed $\omega_{m}$

$L_{e i}$ : $\quad$ Output torque the motor of exciter $i(i=1,2)$

$L_{e 0 i}$ : $\quad$ Output torque the motor of exciter $i(i=1,2)$ at a certain speed $\omega_{m}$

D: $\quad$ Synchronization performance index of the vibrating system 


\section{Data Availability}

All the data used to support the findings of this study are available from the corresponding author upon request.

\section{Conflicts of Interest}

The authors declare that they have no conflicts of interest.

\section{Acknowledgments}

This study was supported by the National Natural Science Foundation of China (Grant nos. 51305070 and 51905081) and Natural Science Foundation of Hebei Province (Grant no. E2019501117).

\section{References}

[1] A. Pikovsky, M. Rosenblum, and J. Kurths, Synchronization: A Universal Concept in Nonlinear Science, Cambridge University Press, London, UK, 2001.

[2] A. K. Kozlov, M. M. Sushchik, Y. I. Molkov, and A. S. Kuznetsov, "Bistable phase synchronization and chaos in a system of coupled van der pol-duffing pscillators," International Journal of Bifurcation and Chaos, vol. 9, no. 12, pp. 2271-2277, 1999.

[3] P. Chaikhan, T. D. Frank, and S. Mongkolsakulvong, "Inphase and anti-phase synchronization in an active Nambu mechanics system," Acta Mechanica, vol. 227, no. 10, pp. 2703-2717, 2016.

[4] R. C. Elson, A. I. Selverston, H. D. I. Abarbanel, and M. I. Rabinovich, "Inhibitory synchronization of bursting in biological neurons: dependence on synaptic time constant," Journal of Neurophysiology, vol. 88, no. 3, pp. 1166-1176, 2002.

[5] K. Suzuki and T. Tsubone, "In-phase and anti-phase synchronization phenomena in coupled systems of piecewise constant oscillators," IEICE Transactions on Fundamentals of Electronics, Communications and Computer Sciences, vol. E98.A, no. 1, pp. 340-353, 2015.

[6] I. I. Blekhman, "About the paper by A. I. Lurie on synchronization theory," IFAC Proceedings Volumes, vol. 34, no. 6, pp. 31-34, 2001.

[7] I. I. Blekhman, "Self-synchronization of vibrators in some types of vibration machines," Inzhenerny Sb, vol. 16, pp. 49-72, 1953.

[8] X. Zhang, B. Wen, and C. Zhao, "Theoretical study on synchronization of two exciters in a nonlinear vibrating system with multiple resonant types," Nonlinear Dynamics, vol. 85, no. 1, pp. 141-154, 2016.

[9] X. Zhang, J. Xu, C. Zhao, and B. Wen, "Synchronization of dual homodromy rotors with eccentric masses in a nonlinear vibrating system," Transactions of the Canadian Society for Mechanical Engineering, vol. 40, no. 3, pp. 303-315, 2016.

[10] P. Fang and Y. Hou, "Synchronization characteristics of a rotor-pendula system in multiple coupling resonant systems," Proceedings of the Institution of Mechanical Engineers, Part C: Journal of Mechanical Engineering Science, vol. 232, no. 10, pp. 1802-1822, 2018.

[11] P. Fang, Y. Hou, and M. Du, "Synchronization behavior of triple-rotor-pendula system in a dual-super-far resonance system," Proceedings of the Institution of Mechanical
Engineers, Part C: Journal of Mechanical Engineering Science, vol. 233, no. 5, pp. 1620-1640, 2019.

[12] L. Li and X. Chen, "Double synchronization states of two exciters with horizontal asymmetric structure in a vibrating system," Journal of Vibroengineering, vol. 19, no. 5, pp. 3883-3894, 2017.

[13] X. Zhang, C. Li, Z. Wang, and S. Cui, "Synchronous stability of four homodromy vibrators in a vibrating system with double resonant types," Shock and Vibration, vol. 2018, Article ID 9641231, 20 pages, 2018.

[14] C. Zhao, H. Zhu, T. Bai, and B. Wen, "Synchronization of two non-identical coupled exciters in a non-resonant vibrating system of linear motion. Part II: numeric analysis," Shock and Vibration, vol. 16, no. 5, pp. 517-528, 2009.

[15] C. Zhao, H. Zhu, R. Wang, and B. Wen, "Synchronization of two non-identical coupled exciters in a non-resonant vibrating system of linear motion. Part I: theoretical analysis," Shock and Vibration, vol. 16, no. 5, pp. 505-515, 2009.

[16] C. Zhao, Q. Zhao, Y. Zhang, and B. Wen, "Synchronization of two non-identical coupled exciters in a non-resonant vibrating system of plane motion," Journal of Mechanical Science and Technology, vol. 25, no. 1, pp. 49-60, 2011.

[17] Y. Li, T. Ren, J. Zhang, and M. Zhang, "Synchronization of two eccentric rotors driven by one motor with two flexible couplings in a spatial vibration system," Mathematical Problems in Engineering, vol. 2019, Article ID 2969687, 13 pages, 2019.

[18] X. Chen, X. Kong, X. Zhang, L. Li, and B. Wen, "On the synchronization of two eccentric rotors with common rotational Axis: theory and experiment," Shock and Vibration, vol. 2016, Article ID 6973597, 14 pages, 2016.

[19] B. Wen, J. Fan, C. Zhao, and W. Xiong, Vibratory Synchronization and Controlled Synchronization in Engineering, Science Press, Beijing, China, 2009.

[20] M. Zou, P. Fang, H. Peng, D. Hou, M. Du, and Y. Hou, "Study on synchronization characteristics for self-synchronous vibration system with dual-frequency and dual-motor excitation," Journal of Mechanical Science and Technology, vol. 33, no. 3, pp. 1065-1078, 2019.

[21] J. Inoue, Y. Araki, and Y. Watanabe, "On the self-synchronization of mechanical vibrators: Part6, multiple synchronization," Transactions of the Japan Society of Mechanical Engineers, vol. 42, no. 353, pp. 111-117, 1976.

[22] L. Jia, X. Kong, J. Zhang, Y. Liu, and B. Wen, "Multiplefrequency controlled synchronization of two homodromy eccentric rotors in a vibratory system," Shock and Vibration, vol. 2018, Article ID 4941357, 12 pages, 2018.

[23] L. Li and X. Chen, "Times-frequency synchronization of two exciters with the opposite rotating directions in a vibration system," Journal of Sound and Vibration, vol. 443, pp. 591-604, 2019.

[24] B. C. Wen, X. L. Zhang, C. Y. Zhao, S. Y. Liu, and X. Y. Lin, "Vibratory synchronization modes and their applications based on a vibrating bedstand with coupled exciters," in Proceedings of the 2015 IFToMM World Congress, National Taiwan University, Taipei, Taiwan, 2015.

[25] C. Zhao, H. Zhu, Y. Zhang, and B. Wen, "Synchronization of two coupled exciters in a vibrating system of spatial motion," Acta Mechanica Sinica, vol. 26, no. 3, pp. 477-493, 2010.

[26] H. K. Khalil, Nonlinear Systems, Prentical Hall, Upper Saddle River, NJ, USA, 3rd edition, 2002.

[27] X.-L. Zhang, B.-C. Wen, and C.-Y. Zhao, "Synchronization of three homodromy coupled exciters in a non-resonant vibrating system of plane motion," Acta Mechanica Sinica, vol. 28, no. 5, pp. 1424-1435, 2012. 
[28] R. A. Horn and C. R. Johnson, Matrix Analysis, Cambridge University Press, London, UK, 2nd edition, 2013.

[29] E. Holzbecher and E. Holzbecher, "Nonlinear systems," in Environment Model., pp. 347-362, Prentice-Hall, Berlin, Germany, 3rd edition, 2012.

[30] R. V. Patel and M. Toda, "Quantitative measures of robustness for multivariable systems," in Jt. Autom. Control Conf., vol. 17, 35 pages, 1980.

[31] X.-L. Zhang, C.-Y. Zhao, and B.-C. Wen, "Theoretical and experimental study on synchronization of the two homodromy exciters in a non-resonant vibrating system," Shock and Vibration, vol. 20, no. 2, pp. 327-340, 2013. 